\title{
Consistent Structural Linearisation in Flexible-Body Dynamics with Large Rigid-Body Motion
}

\author{
Henrik Hesse $^{\mathrm{a}}$, Rafael Palacios ${ }^{\mathrm{a}, *}$ \\ ${ }^{a}$ Department of Aeronautics, Imperial College, London SW7 2AZ, United Kingdom
}

\begin{abstract}
A consistent linearisation, using perturbation methods, is obtained for the structural degrees of freedom of flexible slender bodies with large rigid-body motions. The resulting system preserves all couplings between rigid and elastic motions and can be projected onto a few vibration modes of a reference configuration. This gives equations of motion with cubic terms in the rigid-body degrees of freedom and constant coefficients which can be pre-computed prior to the time-marching simulation. Numerical results are presented to illustrate the approach and to show its advantages with respect to mean-axes approximations.
\end{abstract}

Keywords: flexible-body dynamics, geometrically-nonlinear beam modeling, vibration modes, mean axes

\section{Nomenclature}

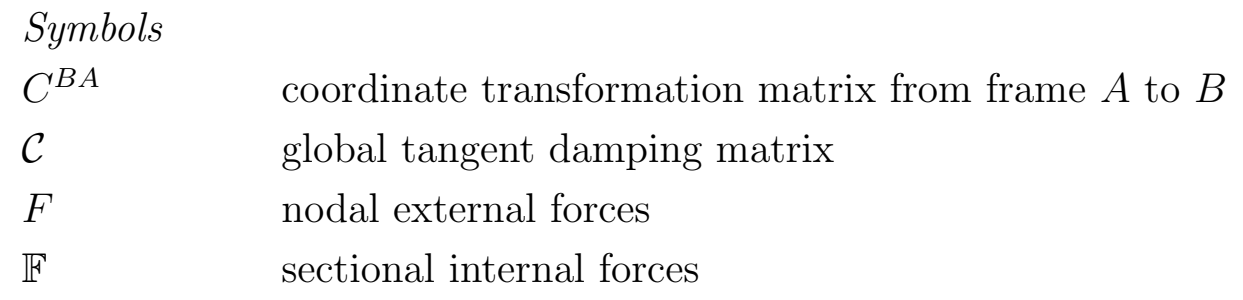

${ }^{*}$ Corresponding author. Address: Room 355, Roderic Hill Building. Tel.: +44 (0)20 75945075

Email address: rpalacio@imperial.ac.uk (Rafael Palacios) 


\begin{tabular}{|c|c|}
\hline$H$ & sectional angular momenta \\
\hline$I$ & unit matrix \\
\hline$K$ & sectional local curvature \\
\hline $\mathcal{K}$ & global tangent stiffness matrix \\
\hline$M$ & nodal external moments \\
\hline $\mathbb{M}$ & sectional internal moments \\
\hline $\mathcal{M}$ & global tangent mass matrix \\
\hline $\mathcal{M}_{c s}$ & cross-sectional mass matrix \\
\hline$N$ & matrix of shape functions \\
\hline$p$ & generalised displacements in modal basis \\
\hline$P$ & sectional translational momenta \\
\hline$Q$ & global vector of generalised forces \\
\hline$r$ & position vector of the origin of body-fixed frame, $A$ \\
\hline$R$ & local position vector along the beam reference line \\
\hline$s$ & arc-length along beam reference line \\
\hline $\mathcal{S}_{c s}$ & cross-sectional stiffness matrix \\
\hline$T$ & tangential operator \\
\hline$v$ & inertial translational velocity of the body-fixed frame, $A$ \\
\hline$V$ & inertial translational velocity at a beam cross section \\
\hline$\beta$ & vector of global translational and rotational velocities \\
\hline$\gamma$ & beam local force strain \\
\hline$\zeta$ & quaternion for global orientation of the body-fixed frame, $A$ \\
\hline$\eta$ & vector of nodal displacements and rotations \\
\hline$\kappa$ & beam local moment strain \\
\hline$\xi$ & beam cross-sectional coordinates \\
\hline$\Phi$ & matrix of mode shapes \\
\hline$\delta \phi$ & global virtual rotations \\
\hline$\delta \Phi$ & local virtual rotations \\
\hline$\Psi$ & local Cartesian Rotation Vector (CRV) along the beam reference line \\
\hline$\omega$ & inertial angular velocity of the body-fixed frame, $A$ \\
\hline$\Omega$ & inertial angular velocity at a beam cross section \\
\hline
\end{tabular}




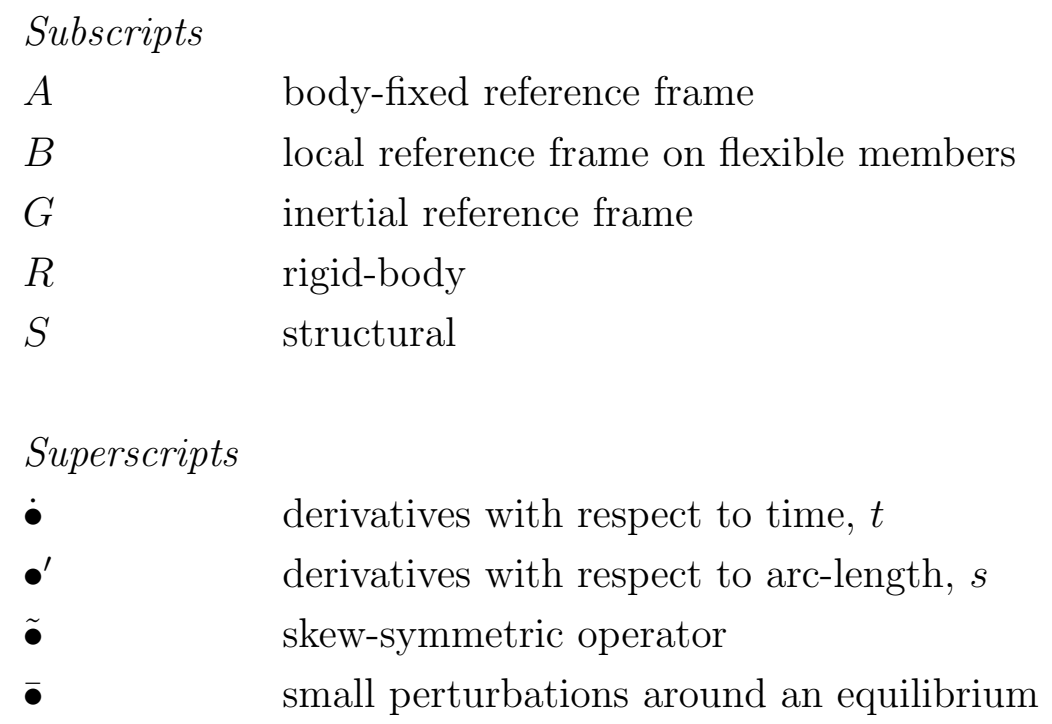

\section{Introduction}

Geometrically-nonlinear beam theories are key in understanding the global static or dynamic response in highly-optimised structures with slender subcomponents. Typical examples include the dynamics of space antennae or the aeroelastic analysis of large off-shore wind turbine blades and helicopter rotor blades. Moreover, an increase in flexibility of load carrying structures has been catalysed by the use of composite materials, which has required a parallel effort in developing geometrically-nonlinear composite beam models. Those are typically based on a two step procedure: first, a process of dimensional reduction (homogenisation) in which the three-dimensional composite structure is reduced to averaged properties along the reference line [1 4], and second the solution of the one-dimensional dynamic equations of motion on the homogenised structure (the composite beam) [5] 8 . The literature on composite beam modelling is quite extensive and it is not the purpose here to present a comprehensive review, which can be found, for instance, in the monograph on the topic by Hodges [9].

The focus here will be on flexible unsupported (free-flying) slender structures with 
very low natural frequencies. This situation has traditionally been studied in the transient dynamic analysis of space structures, but it has found a new application in the development of light UAVs with very high-aspect-ratio wings [10]. On the positive side, the presence of air loads introduces aerodynamic damping (at low enough flight speeds) that helps to stabilize these platforms. However, the higher load levels require a complex aeroelastic balancing to minimise the instantaneous wing loading in highly efficient designs. A better understanding of the effect of large overall rigid-body motion with possibly large elastic deformations on the dynamic response of such structures can improve the design process and is crucial for the design of control systems to optimise their performance [11].

A first approximation to introduce structural dynamics effects into the vehicle dynamics is through the mean-axes assumption [12]. It assumes that there exists an instantaneous reference frame which decouples the structural and rigid-body dynamics degrees of freedom (DoF), thus providing a much simplified description that superimposes elastic deformations to the rigid-body dynamics. This approach has been followed in a number of studies [13 16, mostly for relatively stiff vehicles. For linear undamped systems, it is indeed possible to transform the system equations to a set of decoupled ordinary differential equations due to the orthogonality property of normal modes. However, if the structure is subject to arbitrary motion, which may include gyroscopic effects, the classical modal analysis generally fails to decouple the system equations of motion (EoM). This implies that the mean-axes approximation neglects some coupling terms, even for the linear problem, as structures can generally be subject to gyroscopic motion [17]. Meirovitch and Juang [18, 19] developed a modal analysis for flexible gyroscopic systems which decouples the system EoM for problems where the resulting damping matrix is skew-symmetric. Meirovitch [20] also derived linearised equations about a reference rigid manoeuvre using perturbations on both rigid-body 
and the elastic DoF.

For the case of large structural deformations, nonlinear analysis of space-curved beams have been developed based on Cosserat's model with geometrically-exact kinematics, as written by Love [21] and Reissner [22]. Simo and Vu-Quoc [23, 24] generalised the approach to the fully three-dimensional dynamic case by introducing quaternions to describe the rotational DoF. The equations are solved using finite elements with displacement and finite rotation coordinates as the independent variables. Later, Simo and $\mathrm{Vu}-\mathrm{Quoc}$ extended the theory to rods undergoing large unconstrained motions in space including rotations [25, 26]. They used a global body-fixed reference frame attached to the reference configuration to include the rigid-body motion of the beam. A similar approach was used by Cardona and Géradin [27, 28] using the Cartesian Rotation Vector (CRV) to describe the sectional orientation. Whereas nonlinear theories are capable of capturing arbitrarily-large deformations and rotations, the resulting system of equations is numerically stiff because of large rigid-body variables and comparably-small elastic deformations. This raises the question of the required fidelity of the structural model to capture the elastic deformations efficiently. In many applications, it can be assumed that structural deformations remain small while the vehicle is undergoing large (i.e. nonlinear) rigid-body motion.

This work investigates the consistent structural linearisation of the structural DoF in nonlinear flexible-body dynamics problems with small elastic deformations. That allows the projection of the nonlinear EoM onto the vibration modes of the unconstrained structure to reduce the problem size while keeping the nonlinearity in the rigid-body dynamics equations and the coupling between rigid-body and structural dynamics. At last, we aim to investigate this coupling effect of the nonlinear gyroscopic forces on the elastic deformations to assess the mean-axes approximation. The application in mind is for the nonlinear flight dynamics of flexible aircraft [29] and the methodology 
has been integrated into the framework for Simulation of High-Aspect Ratio Planes (SHARP) [30]. Our starting point will be a geometrically-exact formulation of the beam dynamics, using a displacement-based approach [24, 28]. Local beam rotations are parametrised using the CRV with respect to a body-attached reference frame. The description is finalised with the six DoF rigid-body EoM of that body-attached frame of reference. The elastic DoF of the resulting set of nonlinear EoM are linearised using a perturbation approach. In a second step, the modal projection is done on the elastic modes of the unrestrained system obtained at an arbitrary reference condition. The modal coefficients are then written in constant tensor form with up to cubic terms because of the nonlinear rigid-body velocities, which are sparse and can be pre-computed. Comparison of numerical results obtained from the implemented nonlinear form of the

flexible-body dynamics equations will allow for an evaluation of each assumption made in the linearisation/reduction process. The different methodologies are applied to simple but representative geometries with low-frequency, elastic deformations.

\section{Nonlinear Flexible-Body Dynamics}

A geometrically-exact composite beam model [6, 28] is used to represent the dynamics of very flexible unconstrained structures. As shown in Fig. 1, the deformation of the structure is described in terms of a moving, body-fixed reference coordinate system, $A$, which moves with respect to an inertial (ground) frame, $G$, by the inertial translational and angular velocities, $v_{A}(t)$ and $\omega_{A}(t)$, of its origin. Subscripts are used to indicate the coordinate system in which each vector magnitude is projected. The orientation of the global frame, $A$, with respect to the inertial frame, $G$, is given by the coordinate transformation matrix $C^{G A}(t)$. The local orientation of each beam cross-section is defined by the local coordinate system, $B$, in the current configuration. 


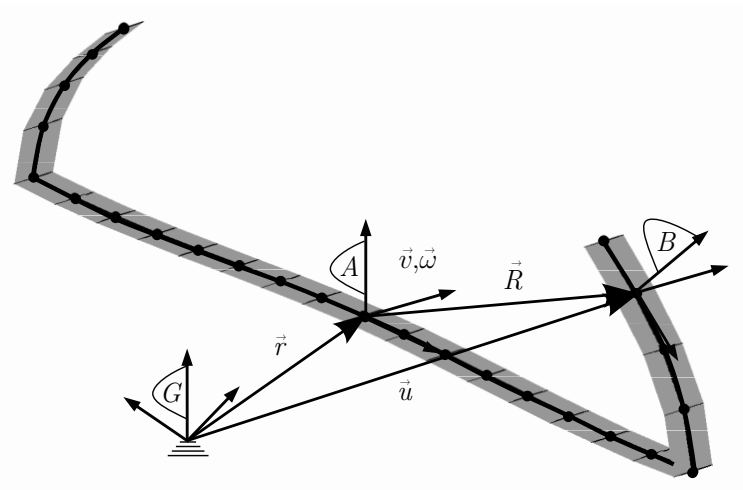

Figure 1: Multi-beam configuration with the definition of reference frames.

The relative orientation between the global body-fixed reference frame, $A$, and the local deformed frame, $B$, is parametrised by the CRV, $\Psi(s, t)$. The corresponding coordinate transformation matrix is $C^{B A}(s, t)=C(\Psi)$. The nodal positions, $R_{A}(s, t)$, expressed in the body-fixed frame, $A$, and the cross-sectional orientations, $\Psi(s, t)$, form the independent set of variables in this formulation. The deformation of the reference line going from the undeformed state at $t=0$ to the current state at time $t$ is given by the force and moment strains [6], which are written in terms of the CRV as

$$
\begin{aligned}
& \gamma(s, t)=C(\Psi(s, t)) R_{A}^{\prime}(s, t)-C(\Psi(s, 0)) R_{A}^{\prime}(s, 0), \\
& \kappa(s, t)=T(\Psi(s, t)) \Psi^{\prime}(s, t)-T(\Psi(s, 0)) \Psi^{\prime}(s, 0) .
\end{aligned}
$$

where $(\bullet)^{\prime}$ is the derivative with respect to the arc-length $s$, and $T(\Psi)$ is the tangential operator. Its definition can be found, for instance, in [28]. The local curvature is obtained as $K_{B}=T(\Psi) \Psi^{\prime}$.

The inertial properties will be determined by its translational and angular inertial velocities at each location defined by the arc-length $s$, given, respectively, as

$$
\begin{aligned}
& V_{B}(s, t)=C(\Psi(s, t)) \dot{R}_{A}(s, t)+C(\Psi(s, t))\left[v_{A}(t)+\widetilde{\omega}_{A}(t) R_{A}(s, t)\right], \\
& \Omega_{B}(s, t)=T(\Psi(s, t)) \dot{\Psi}(s, t)+C(\Psi(s, t)) \omega_{A}(t)
\end{aligned}
$$


where $(\tilde{\bullet})$ is the cross-product (or skew-symmetric) operator. The above expression can also be expressed in compact matrix form as

$$
\left\{\begin{array}{c}
V_{B} \\
\Omega_{B}
\end{array}\right\}=\Lambda\left\{\begin{array}{c}
\dot{R}_{A} \\
\dot{\Psi}
\end{array}\right\}+A_{R C}\left\{\begin{array}{c}
v_{A} \\
\omega_{A}
\end{array}\right\} .
$$

The dynamics of the beam in a given time interval $\left[t_{1}, t_{2}\right]$ is to be analysed in the (moving) body-attached reference frame $A$. From Hamilton's principle, it is

$$
\int_{t_{1}}^{t_{2}} \int_{\Gamma}[\delta \mathcal{T}-\delta \mathcal{U}+\delta \mathcal{W}] d s d t=0
$$

where $\Gamma$ refers to the total length of all reference lines. In this expression, $\mathcal{T}$ and $\mathcal{U}$ are the kinetic and internal energy densities per unit length, respectively, and $\delta \mathcal{W}$ is the virtual work of applied loads per unit length. The virtual strain and kinetic energies are written as [6]

$$
\begin{aligned}
\delta \mathcal{U} & =\left[\begin{array}{ll}
\delta \gamma^{\top} & \delta \kappa^{\top}
\end{array}\right] \mathcal{S}_{c s}\left[\begin{array}{ll}
\gamma^{\top} & \kappa^{\top}
\end{array}\right]^{\top}=\left[\begin{array}{ll}
\delta \gamma^{\top} & \delta \kappa^{\top}
\end{array}\right]\left[\begin{array}{ll}
\mathbb{F}_{B}^{\top} & \mathbb{M}_{B}^{\top}
\end{array}\right]^{\top}, \\
\delta \mathcal{T} & =\left[\begin{array}{ll}
\delta V_{B}^{\top} & \delta \Omega_{B}^{\top}
\end{array}\right] \mathcal{M}_{c s}\left[\begin{array}{ll}
V_{B}^{\top} & \Omega_{B}^{\top}
\end{array}\right]^{\top}=\left[\begin{array}{ll}
\delta V_{B}^{\top} & \delta \Omega_{B}^{\top}
\end{array}\right]\left[\begin{array}{ll}
P_{B}^{\top} & H_{B}^{\top}
\end{array}\right]^{\top},
\end{aligned}
$$

where we have introduced the internal forces and moments, $\mathbb{F}_{B}$ and $\mathbb{M}_{B}$, and the local translational and angular momenta, $P_{B}$ and $H_{B}$, respectively. The fully-populated $6 \times 6$ cross-sectional mass and stiffness matrices, $\mathcal{M}_{c s}$ and $\mathcal{S}_{c s}$, can be obtained through an appropriate cross-sectional analysis methodology [3]. Finally, all virtual magnitudes are expressed in terms of the independent set of variables, through the kinematic relations introduced in Eqs. (1) and (2).

Consider next the applied volume (or surface) forces $\mu_{G}$ acting on the beam sections. The corresponding virtual work per unit length is $\delta \mathcal{W}=\left\langle\delta X_{G}^{\top} \mu_{G}\right\rangle$, where $\delta X_{G}$ is the virtual position vector in the current configuration at the material points where forces $\mu_{G}$ are applied, and $\langle\bullet\rangle$ is the integral over the area (or area contour) of the local cross 
section. If $\xi_{B}$ are the local cross-sectional coordinates, the position vector itself is

$$
X_{G}=r_{G}+C^{G A} R_{A}+C^{G A} C^{\top} \xi_{B},
$$

and the virtual work per unit length is then

$$
\delta \mathcal{W}=\left[\delta r_{G}^{\top} C^{G A}+\delta R_{A}^{\top}+\delta \phi_{A}^{\top}\left(\tilde{R}_{A}+C^{\top} \tilde{\xi}_{B} C\right)\right] C^{\top} F_{B}+\delta \Phi_{B}^{\top} M_{B}
$$

where $\delta \tilde{\phi}_{A}=C^{A G} \delta C^{G A}$ and $\delta \tilde{\Phi}_{B}=C \delta C^{\top}$ are, respectively, the global and local virtual rotations. The set of resultant forces and moments per unit beam length has been defined as $F_{B}=\left\langle\mu_{B}\right\rangle$ and $M_{B}=\left\langle\tilde{\xi}_{B} \mu_{B}\right\rangle$, respectively. To obtain the EoM, we approximate both the position vector, $R_{A}$, and the CRV, $\Psi$, using a finite-element discretisation (here, 2- and 3-noded elements, corresponding to linear and quadratic interpolation, have been implemented). There are known issues with objectivity of the interpolation operation of finite rotations [31, 32], however, good performance of the implementation is observed for quadratic elements or fine enough discretisation with linear elements. If $\eta$ is the column vector with all the nodal displacements and rotations and $\beta^{\top}=\left\{\begin{array}{ll}v_{A}^{\top} & \omega_{A}^{\top}\end{array}\right\}$, the discrete form of the dynamic equations is written as

$$
\mathcal{M}(\eta)\left\{\begin{array}{c}
\ddot{\eta} \\
\dot{\beta}
\end{array}\right\}+\left\{\begin{array}{c}
Q_{g y r}^{S} \\
Q_{g y r}^{R}
\end{array}\right\}+\left\{\begin{array}{c}
Q_{s t i f}^{S} \\
0
\end{array}\right\}=\left\{\begin{array}{c}
Q_{e x t}^{S} \\
Q_{e x t}^{R}
\end{array}\right\},
$$

where structural and rigid-body components (denoted by superscripts $S$ and $R$ ) have been identified in the gyroscopic, stiffness and external forces. If $N(s)$ is the matrix of shape functions for all nodes, those discrete generalised forces are written as

$$
\begin{aligned}
Q_{s t i f}^{S}(\eta) & =\int_{\Gamma}\left(N^{\top} \Upsilon^{\top} A_{K}+N^{\top} \Upsilon^{\prime \top}+N^{\prime \top} \Lambda^{\top}\right) \hat{\mathbb{F}} d s, \\
Q_{g y r}(\eta, \dot{\eta}, \beta) & =\int_{\Gamma}\left[\begin{array}{ll}
\Lambda N & A_{R C}
\end{array}\right]^{\top}\left(\mathcal{M}_{c s} \hat{V}_{g y r}+A_{V \Omega} \mathcal{M}_{c s} \hat{V}\right) d s, \\
Q_{e x t}(\eta, \dot{\eta}, \beta, \zeta) & =\int_{\Gamma}\left[\begin{array}{ll}
\Lambda N & A_{R C}
\end{array}\right]^{\top} \hat{F} d s,
\end{aligned}
$$


where $\zeta$ is the orientation of the body-fixed reference system, which is obtained from integration of its angular velocity, $\omega_{A}$ [33]. Also, the following variables for resultant loads, local inertial velocities, and internal forces have been defined to simplify notation,

$$
\hat{F}=\left\{\begin{array}{l}
F_{B} \\
M_{B}
\end{array}\right\}, \hat{V}=\left\{\begin{array}{c}
V_{B} \\
\Omega_{B}
\end{array}\right\}, \hat{\mathbb{F}}=\left\{\begin{array}{c}
\mathbb{F}_{B} \\
\mathbb{M}_{B}
\end{array}\right\} .
$$

The gyroscopic velocities $\hat{V}_{\text {gyr }}$ in the second of Eq. (9) are defined as

$$
\hat{V}_{g y r}(\eta, \dot{\eta}, \beta)=\left\{\begin{array}{c}
V_{g y r} \\
\Omega_{g y r}
\end{array}\right\}=\left\{\begin{array}{c}
C \tilde{\omega}_{A} \dot{R}_{A}+\tilde{V}_{B} T \dot{\Psi} \\
\dot{T} \dot{\Psi}+\tilde{\Omega}_{B} T \dot{\Psi}
\end{array}\right\}
$$

and the following $6 \times 6$ matrix operators were used in the definition of the discrete generalised forces,

$$
\Upsilon=\left[\begin{array}{cc}
I & 0 \\
0 & T
\end{array}\right], A_{K}=\left[\begin{array}{cc}
0 & 0 \\
-\left(\tilde{e}_{1}+\tilde{\gamma}\right) & -\tilde{K}_{B}
\end{array}\right], A_{V \Omega}=\left[\begin{array}{cc}
\tilde{\Omega}_{B} & 0 \\
\tilde{V}_{B} & \tilde{\Omega}_{B}
\end{array}\right] .
$$

The tangent mass matrices have been defined as a function of the deformed state, $\eta$, as

$$
\mathcal{M}(\eta)=\left[\begin{array}{ll}
\mathcal{M}^{S S} & \mathcal{M}^{S R} \\
\mathcal{M}^{R S} & \mathcal{M}^{R R}
\end{array}\right]=\int_{\Gamma}\left[\begin{array}{ll}
\Lambda N & A_{R C}
\end{array}\right]^{\top} \mathcal{M}_{c s}\left[\begin{array}{ll}
\Lambda N & A_{R C}
\end{array}\right]
$$

where the operators $\Lambda$ and $A_{R C}$ have been defined in Eq. (3). The nonlinear secondorder differential equations of Eq. (8), couples the geometrically-nonlinear beam dynamics with the nonlinear rigid-body motion of the flexible body through the inertial and gyroscopic forcing terms. Equation (8) may need to be solved together with the propagation equations that determine the position and orientation of the body-fixed reference frame. Those can be found, for instance, in Ref. [33]. The resulting ordinary differential equations can be solved by any standard time-integration method [34 37]. An implicit Newmark- $\beta$ integration scheme was used in all numerical examples in this work. 


\section{Linearisation of Elastic Degrees of Freedom in Nonlinear Flexible-Body Dynamics}

We assume now that the structural DoF are small, while the rigid-body DoF are arbitrarily large. This linear-flexible/nonlinear-rigid formulation will form the basis for the subsequent modal projection of the EoM in section 3.3 .

\subsection{Perturbation of Structural Dynamic Equations}

Equation (8) can be linearised only in the elastic DoF by means of a perturbation approach. The (large) elastic deformations at the static equilibrium condition will be given by $\eta$, while $\bar{\eta}$ will be the small elastic deformations that occur together with the

(not-necessarily small) rigid-body velocities $\beta$. Perturbation of the elastic DoF on the nonlinear EoM gives linear inertial and elastic terms, which depend on $\eta$ only. This results in the definition of the tangent mass matrix, given in Eq. (13), and the contribution of elastic forces to the tangent stiffness matrix, $\mathcal{K}_{\text {stif }}^{S}$, respectively. Perturbation of the gyroscopic forces will require particular attention because of the coupling between rigid-body and elastic states. Partial linearisation of the gyroscopic forces results in updated expressions for the gyroscopic damping and stiffness matrices in Eq. 222.

\subsubsection{Elastic Perturbations of Beam Kinematics}

Perturbations of the elastic DoF partially linearise the local translational and angular inertial velocities, introduced in Eq. (2), which can be expressed as

$$
\hat{V}(\eta, \bar{\eta}, \dot{\bar{\eta}}, \beta)=\left\{\begin{array}{l}
V_{B}^{R} \\
\Omega_{B}^{R}
\end{array}\right\}+\left\{\begin{array}{l}
\bar{V}_{B} \\
\bar{\Omega}_{B}
\end{array}\right\}+\text { H.O.T. }
$$

where the rigid-body and elastic contributions to the local velocities are, respectively,

$$
\left\{\begin{array}{c}
V_{B}^{R} \\
\Omega_{B}^{R}
\end{array}\right\}=A_{R C} \beta,\left\{\begin{array}{l}
\bar{V}_{B} \\
\bar{\Omega}_{B}
\end{array}\right\}=\Lambda \dot{\bar{\eta}}-\left(A_{V \Omega}^{R}\right)^{\top} \Lambda \bar{\eta} .
$$


Additionally, the gyroscopic velocities defined in Eq. (11) are approximated as

$$
\hat{V}_{g y r}(\eta, \bar{\eta}, \beta)=\left\{\begin{array}{c}
\bar{V}_{g y r} \\
\bar{\Omega}_{g y r}
\end{array}\right\}=-\left(A_{V \Omega}^{R}\right)^{\top} \Lambda \bar{\eta}+\text { H.O.T. }
$$

with

$$
A_{V \Omega}^{R}=\left[\begin{array}{cc}
\tilde{\Omega}_{B}^{R} & 0 \\
\tilde{V}_{B}^{R} & \tilde{\Omega}_{B}^{R}
\end{array}\right] .
$$

\subsubsection{Elastic Perturbations of Gyroscopic Forces}

It is now possible to derive expressions for the contribution of the gyroscopic forces to damping and stiffness matrices, such that the perturbed gyroscopic forces can be expressed in the form of

$$
Q_{g y r}(\eta, \bar{\eta}, \dot{\bar{\eta}}, \beta)=\left[\begin{array}{ll}
\overline{\mathcal{C}}^{S S} & \overline{\mathcal{C}}^{S R} \\
\overline{\mathcal{C}}^{R S} & \overline{\mathcal{C}}^{R R}
\end{array}\right]\left\{\begin{array}{l}
\dot{\bar{\eta}} \\
\beta
\end{array}\right\}+\left[\begin{array}{ll}
\overline{\mathcal{K}}_{g y r}^{S} & 0 \\
\overline{\mathcal{K}}_{g y r}^{R} & 0
\end{array}\right]\left\{\begin{array}{c}
\bar{\eta} \\
0
\end{array}\right\}+\text { H.O.T. }
$$

with the components of the gyroscopic damping and stiffness matrices defined as

$$
\begin{aligned}
\overline{\mathcal{C}}^{S S}(\eta, \beta) & =\int_{\Gamma} N^{\top} \Lambda^{\top}\left[A_{\partial \Omega}^{R} \mathcal{M}_{c s}-\mathcal{M}_{c s}\left(A_{V \Omega}^{R}\right)^{\top}\right] \Lambda N d s, \\
\overline{\mathcal{C}}^{S R}(\eta, \beta) & =\int_{\Gamma} A_{R C}^{\top}\left[A_{\partial \Omega}^{R} \mathcal{M}_{c s}-\mathcal{M}_{c s}\left(A_{V \Omega}^{R}\right)^{\top}\right] \Lambda N d s, \\
\overline{\mathcal{C}}^{R S}(\eta, \beta) & =\int_{\Gamma} N^{\top} \Lambda^{\top} A_{V \Omega}^{R} \mathcal{M}_{c s} A_{R C} d s, \\
\overline{\mathcal{C}}^{R R}(\eta, \beta) & =\int_{\Gamma} A_{R C}^{\top} A_{V \Omega}^{R} \mathcal{M}_{c s} A_{R C} d s, \\
\overline{\mathcal{K}}_{g y r}^{S}(\eta, \beta) & =\int_{\Gamma} N^{\top}\left[\bar{\Lambda}\left(A_{V \Omega}^{R} \hat{P^{R}}\right)-\Lambda^{\top} A_{\partial \Omega}^{R} \mathcal{M}_{c s}\left(A_{V \Omega}^{R}\right)^{\top} \Lambda\right] N d s, \\
\overline{\mathcal{K}}_{g y r}^{R}(\eta, \beta) & =\int_{\Gamma} A_{R C}^{\top} A_{V \Omega}^{R} \mathcal{M}_{c s} B_{\partial \Omega}^{R} \Lambda N d s .
\end{aligned}
$$

Writing the gyroscopic forces in this matrix form illustrates the coupling between the small elastic deformations and the arbitrarily-large rigid-body motions. The following 
$6 \times 6$ matrix operators were used in the above expressions

$$
\begin{aligned}
& \bar{\Lambda}(\eta, \dot{\eta}, \beta, \hat{p})=\left[\begin{array}{cc}
0 & -C^{\top} \tilde{p} T \\
0 & -A_{1}(-\Psi, h)
\end{array}\right], A_{P H}^{R}(\eta, \beta)=\left[\begin{array}{cc}
0 & \widetilde{P}_{B}^{R} \\
\widetilde{P}_{B}^{R} & \widetilde{H}_{B}^{R}
\end{array}\right], \\
& A_{\partial \Omega}^{R}(\eta, \beta)=A_{V \Omega}^{R}-A_{P H}^{R} \mathcal{M}_{c s}^{-1} \\
& B_{\partial \Omega}^{R}(\eta, \beta)=-\left(\left(A_{V \Omega}^{R}\right)^{\top}+\mathcal{M}_{c s}^{-1} A_{P H}^{R}\right),
\end{aligned}
$$

where $P_{B}^{R}$ and $H_{B}^{R}$ are defined as in Eq. (5) and $\hat{p}=\left[\begin{array}{ll}p^{\top} & h^{\top}\end{array}\right]^{\top}$, with $p$ and $h$ being arbitrary $3 \mathrm{x} 1$ column matrices. The matrix operator $A_{1}(\Psi, h)$ is derived from linearisation of the tangential operator, $T(\Psi)$, and it is defined as in Ref. [28]. From Eq. (19], it is clear that the perturbed gyroscopic damping and stiffness matrices are functions of the initial deformation, $\eta$, but also of the instantaneous rigid-body velocity, $\beta$, with linear and quadratic dependencies in the rigid-body DoF.

\subsubsection{Perturbation Equations of Motion}

Finally, perturbation of the elastic DoF around the equilibrium, $\eta$, on the nonlinear EoM, Eq. (8), leads to an expression of the form

$$
\mathcal{M}(\eta)\left\{\begin{array}{l}
\ddot{\bar{\eta}} \\
\dot{\beta}
\end{array}\right\}+\overline{\mathcal{C}}(\eta, \beta)\left\{\begin{array}{l}
\dot{\bar{\eta}} \\
\beta
\end{array}\right\}+\overline{\mathcal{K}}(\eta, \beta)\left\{\begin{array}{l}
\bar{\eta} \\
0
\end{array}\right\}=Q_{\text {ext }}(\bar{\eta}, \dot{\bar{\eta}}, \beta, \zeta)+\text { H.O.T. }
$$

where the expressions of the gyroscopic damping and stiffness matrices in Eq. (19) are substituted in the definition of the perturbed gyroscopic forces, Eq. (18), to obtain $\overline{\mathcal{C}}$ and $\overline{\mathcal{K}}$, respectively. As discussed above, matrices $\overline{\mathcal{C}}$ and $\overline{\mathcal{K}}$ are functions of the rigidbody velocities with linear and quadratic dependencies of $\beta$, respectively. In the next sections, a reduction of the system size will be obtained by projecting the structural variables onto the natural vibration modes of a reference linear system. 


\subsection{Fully-Linearised Flexible-Body Dynamics}

Linearisation of the full dynamic system will be done around a static equilibrium condition, $Q_{s t i f}(\eta)=Q_{e x t}(\eta, \zeta)$, with possibly large elastic deformations, $\eta$, at the reference condition. Small changes from this state will be represented with over-bars, that is, $(\bar{\eta}, \dot{\bar{\eta}}, \ddot{\bar{\eta}}, \bar{\beta}, \dot{\bar{\beta}}, \bar{\zeta})$. For the general case, the linearised (incremental) form of Eq. (8) around a given point is

$$
\mathcal{M}(\eta)\left\{\begin{array}{c}
\ddot{\bar{\eta}} \\
\overline{\bar{\beta}}
\end{array}\right\}+\mathcal{K}(\eta, \beta)\left\{\begin{array}{l}
\bar{\eta} \\
0
\end{array}\right\}=\bar{Q}_{\text {ext }}(\bar{\eta}, \dot{\bar{\eta}}, \bar{\beta}, \bar{\zeta}),
$$

where the mass matrix, $\mathcal{M}$, was defined in Eq. (13). The constant tangent stiffness matrix, $\mathcal{K}$, is obtained through direct linearisation of the discretised elastic forces. The resulting set of coupled linear EoM now forms the basis for aeroelastic stability analyses.

\subsection{Modal Projection of the Perturbed Equations}

Under the assumption of small elastic deformations, it is possible to write the nonlinear system EoM, Eq. (21), in terms of global shape functions by projecting the dynamics equations on the modes of the unconstrained structure. These vibration modes are obtained from the unforced fully-linearised EoM, Eq. (22), such that

$$
\left\{\begin{array}{c}
\bar{\eta} \\
\beta
\end{array}\right\}=\Phi\left\{\begin{array}{c}
p \\
\beta^{*}
\end{array}\right\},
$$

where $p$ is the vector of the projected modal coordinates and $\Phi$ is the matrix of the corresponding mode shapes, which include the six rigid-body modes and the remaining elastic mode shapes describe the motion of the body-fixed frame, $A$, and the elastic deformation with respect to this moving frame. Hence, the vector of modal rigid-body velocities, $\beta^{*}$, is a linear map of the velocities of the body-fixed frame, $A$, and the motion of this frame due to the elastic mode shapes. The coupled EoM can then be 
written in terms of the modal basis, obtained for this system, as

$$
\Phi^{\top} \mathcal{M}(\eta) \Phi\left\{\begin{array}{c}
\ddot{p} \\
\dot{\beta}^{*}
\end{array}\right\}+\Phi^{\top} \overline{\mathcal{C}}(\eta, \beta) \Phi\left\{\begin{array}{c}
\dot{p} \\
\beta^{*}
\end{array}\right\}+\Phi^{\top} \overline{\mathcal{K}}(\eta, \beta) \Phi\left\{\begin{array}{l}
p \\
0
\end{array}\right\}=\Phi^{\top} Q_{\text {ext }}(\bar{\eta}, \dot{\bar{\eta}}, \beta, \zeta)
$$

where the modal damping and stiffness matrices remain functions of the arbitrarilylarge rigid-body DoF and would need to be recomputed for every $\beta$. If we make use of the fact that the damping matrix $\overline{\mathcal{C}}$ and stiffness matrix $\overline{\mathcal{K}}$ have, respectively, linear and quadratic dependencies with the rigid-body DoF, as can be seen from Eq. (19), then it is possible to write the modal matrices in terms of third and fourth-order tensors such that

$$
\begin{aligned}
\Phi_{i j} \overline{\mathcal{C}}_{j k} \Phi_{k l} & =c_{i l r} \beta_{r}(t), \\
\Phi_{i j} \overline{\mathcal{K}}_{j k} \Phi_{k l} & =k_{i l}^{\text {stif }}+k_{i l r s}^{\text {gyr }} \beta_{r}(t) \beta_{s}(t),
\end{aligned}
$$

where the modal stiffness matrix is separated into contributions from linearisation of the elastic forces and secondly from the gyroscopic forces. The tensors $c$ and $k$ are constant in time and their dimensions are $i, l=\{1, \ldots, m\}$ and $r, s=\{1, \ldots, 6\}$ for $m$ number of modes used in the expansion. This approach reduces the size of the structural subsystem dramatically while keeping the rigid-body DoF nonlinear.

Since the only nonlinear terms in Eq. (24) are from the rigid-body dynamics, one should expect to reach similar equations starting from other descriptions of the unsupported beam dynamics, including Total Lagrangian approaches [38].

\section{Numerical Studies}

Previous work [30, 39] has presented verification studies of the current formulation for nonlinear static and dynamic solutions of multi-beam configurations including aerodynamics. The effort in this work will be on unsupported configurations. As there are few results available in the literature for very flexible free-free beams, this work will 
first collate the existing results and will then contribute with additional relevant reference test cases. The linear-flexible/nonlinear-rigid dynamics formulation, introduced in Section 3.1, is exercised on them to explore the performance of the approximation. Finally, we include modes into the problem to explore how the linear-flexible/nonlinearrigid system can be reduced. Those results will finally serve to evaluate the validity of the mean-axes approximation. There are no gravitational or aerodynamic forces acting on the structures in this work. The $L^{2}$ relative error norm, defined as

$$
\varepsilon=\left\|X_{\text {com }}-X_{r e f}\right\| /\left\|X_{r e f}\right\|,
$$

will be used to compare computed results, $X_{\text {com }}$, with a reference solution, $X_{r e f}$.

\subsection{Flying Flexible Beam (FFB)}

Geometry and material properties:

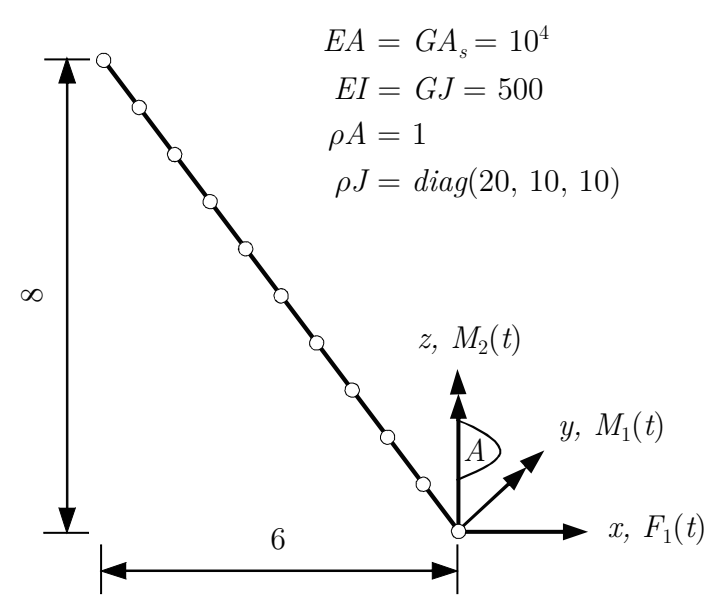

Load history for 2D problem:

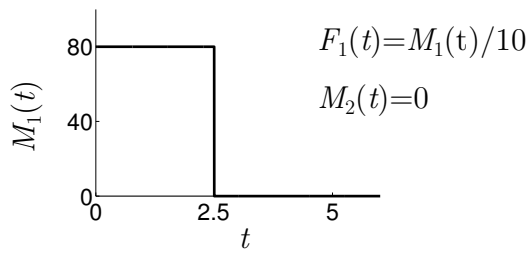

Load history for 3D problem:

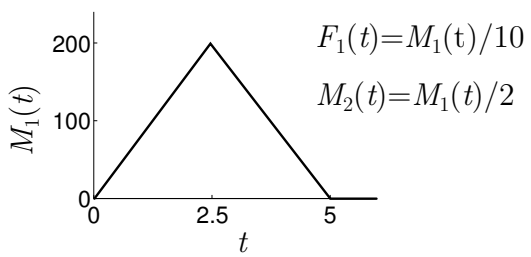

Figure 2: FFB geometry, material properties and load histories [25, 26].

In this section the dynamics of a flexible free-free beam undergoing large overall motion is analysed. The problem at hand was first introduced by Simo and Vu-Quoc for 
the planar case [25], later expanded to 3-D in Ref. [26], and has been used subsequently to validate various geometrically-nonlinear flexible-body dynamics formulations [40,43]. The unconstrained, inclined beam, shown in Fig. 2, is subjected to a set of dead forces and moments acting at the origin of the body-fixed reference frame with the load history also indicated in Fig. 2 for the planar and 3-D case. The beam is discretised using 10 2-noded elements, following Refs. [25, 26].

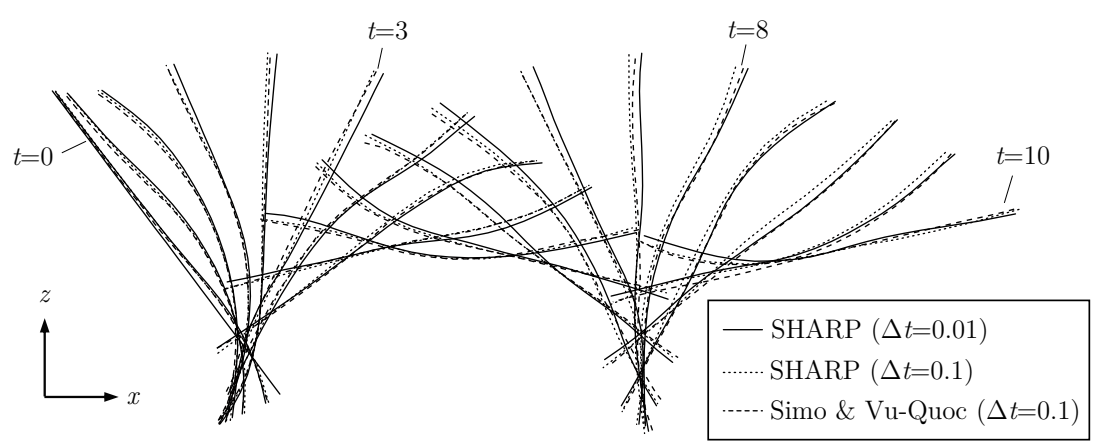

Figure 3: Snapshots of deformed shapes for the 2-D FFB problem in increments of 0.5 for different time steps $\Delta t$. [10 2-noded elements]

The motion of the FFB for the planar case is shown in Fig. 3 for two different time steps, where $\Delta t=0.1$ corresponds to the original simulation properties used in Ref. [25]. However, a smaller time step of $\Delta t=0.01$ is necessary to obtain a converged solution. Results show very good comparison with Simo and Vu-Quoc [25] for matching time steps of $\Delta t=0.1$, but differences can be observed for the converged solution, shown as solid lines in Fig. 3. Frequency domain analysis of the response suggests that the smaller time step of $\Delta t=0.01$ is indeed needed to capture frequencies up to the fourth bending mode which are excited by the impulsive excitation.

In the $3-\mathrm{D}$ case, the FFB is subjected to an additional dead moment $M_{2}(t)$ acting along the $z$ axis, indicated in Fig. 2, to cause out-of-plane motion. Figure 4 shows 


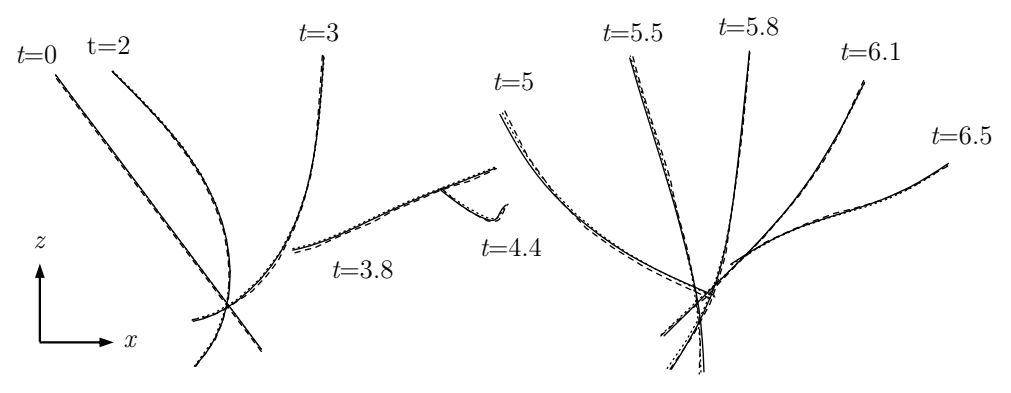

(a) $x-z$ plane

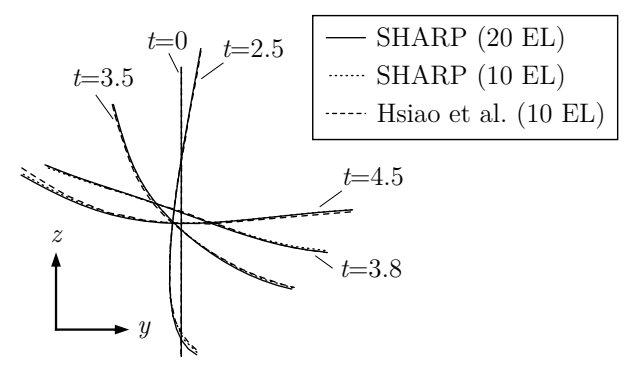

(b) $y$ - $z$ plane

Figure 4: Snapshots of deformed shapes for the 3-D FFB problem with different number of elements. $[\Delta t=0.01]$

the resulting motion for 20 2-noded elements and a time step of $\Delta t=0.01$ for the converged solution. Comparison with the original results published by Simo and VuQuoc [26] shows large discrepancies (not shown here), and the FFB in Ref. [26] appears to be much stiffer than in the results presented in Fig. 4. (A good match with Ref. [26] was achieved for an increased stiffness of $E I_{\alpha}=G J=10^{3}$ ). Instead, for the given material properties in Fig. 2 very good comparison was found with the solution by Hsiao et al. [42] who also reported differences to Ref. [26]. Ref. [42] used a co-rotational finite-element formulation with 10 beam elements. The comparison is shown in Fig. 4 .

Finally, since the FFB is subject to spatially-fixed forces and moments, the motion of the beam centre of mass $(\mathrm{CM})$ becomes a rigid-body dynamics problem which can be 
solved analytically. A convergence study on the $L^{2}$ relative error norm of CM positions, showed that a time step of $\Delta t=0.01$ gives an error below $1 \%$ up to $t=50$. The motion of the FFB for that time step is shown in Fig. 5, and indicates a CM moving along the $x$ direction.

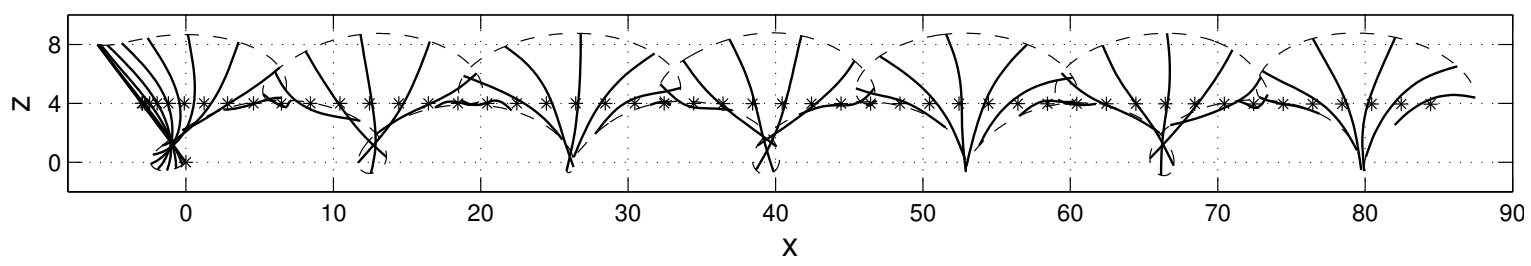

Figure 5: Snapshots in the $x-z$ plane of deformed shapes for the 3 -D FFB problem (solid lines), including positions of both tips (dashed lines) and CM positions (stars) until $t=20$ in increments of 0.4. [ $\Delta t=0.01$ and 20 2-noded elements]

\subsection{Flexible Multi-Beam (FMB) Configuration}

The dynamic response of an unsupported frame is analysed next. This problem will then be used to exemplify the consistent structural linearisation introduced above and to study the application of the mean-axes approach. The geometry and material properties of the FMB are presented in Fig. 6 with different load cases defined in Table 2. The chosen configuration results in a multi-beam problem with shared nodes and large initial rotations. To obtain an acceptable interpolation of the rotation vector, 120 2-noded elements were used. All simulations in this section are for a time step of $\Delta t=0.01$ s. The constant-acceleration Newmark scheme was modified as in Ref. [36] to include a numerical damping of 0.01 that filters some high-frequency oscillations. For a discussion on the damping of the Newmark method and alternative approaches, please see Ref. [44]. 
Material properties:

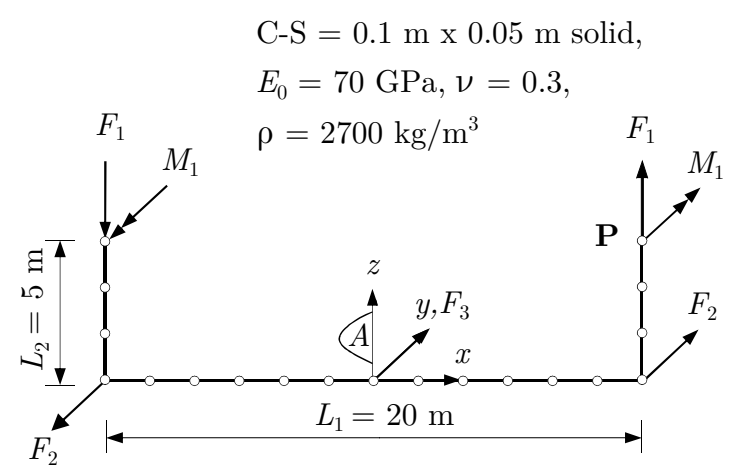

Load history for 3D problem:

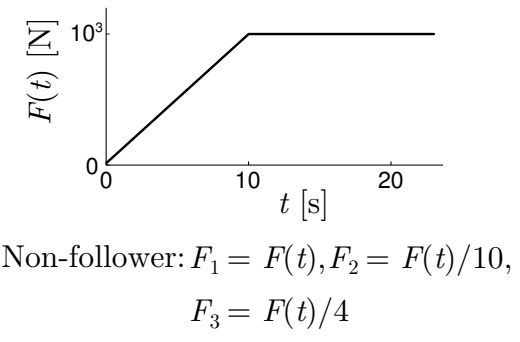

Follower: $M_{1}=L_{1} F(t) / 10$

Figure 6: Definition of the FMB problem.

\subsubsection{Verification of the Nonlinear Model}

SAMCEF Mecano is first used for comparison of results in this numerical study. It is a finite-element solver for nonlinear flexible multi-body dynamics problems based on the formulation by Géradin and Cardona [28]. A variety of forces and moments, load case 1 in Table 2, is applied here to verify the results in terms of 3-D geometrically-nonlinear deformations with nonlinear rigid-body motions subject to Coriolis and centrifugal effects which augment the coupling between structural and rigid-body dynamics.

Table 2: Load cases for the FMB problem.

\begin{tabular}{ccccc}
\hline Load case & $F_{1}$ & $F_{2}$ & $F_{3}$ & $M_{1}$ \\
\hline 1 & $\mathrm{x}$ & $\mathrm{x}$ & $\mathrm{x}$ & $\mathrm{x}$ \\
2 & $\mathrm{x}$ & $\mathrm{x}$ & & \\
\hline
\end{tabular}

The response of the FMB is shown in Figs. 7 and 8 for a time step of $\Delta t=0.01$ s. First, the sequence of motion in Fig. 7 clearly shows that the structure is subject to large deformations. The simultaneous rigid-body translations and rotations increase 


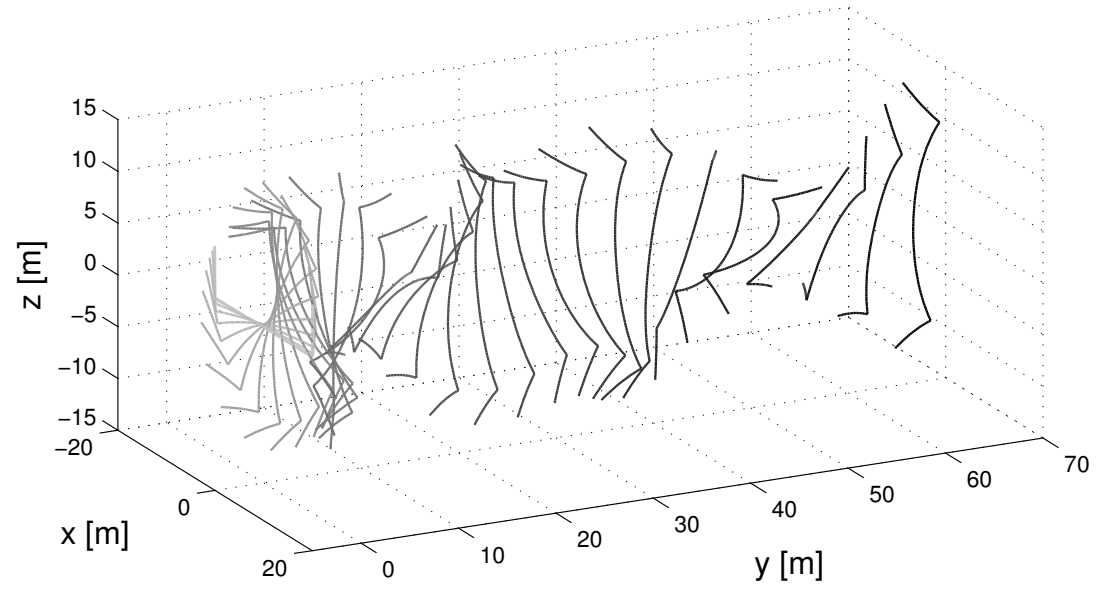

Figure 7: Snapshots of deformed shapes for the FMB, load case 1, at consecutive steps from $t=0 \mathrm{~s}$ (grey) to $t=20 \mathrm{~s}$ (black) in increments of $0.5 \mathrm{~s}$.

the effect of gyroscopic forces on structural and rigid-body dynamics. Comparison of tip positions and rigid-body velocities in Fig. 8 show very good agreement with results obtained in SAMCEF. All quantities are expressed in terms of an inertial frame $G$, initially aligned with frame $A$ in Fig. 1 .

\subsubsection{Structural Linearisation in the Flexible-Body Dynamics of the FMB}

Test case FMB is now subjected to load case 2 in Table 2, which results in 3-D, nonlinear, predominantly rotational, rigid-body motion. The stiffness parameter $\sigma$ is introduced to vary the elastic modulus of the material, such that $E=\sigma E_{0}$. The rigidbody response is presented in Fig. 9 for $\sigma=10$. Figure 10a shows the time history of the vertical tip deformation of point $\mathrm{P}$, normalised with the arm length $L_{2}$, defined in Fig. 6, for varying stiffness. The maximum value of $\max \left(\Delta R_{A z} / L_{2}\right)$, is used to quantify the degree of flexibility and Fig. $10 \mathrm{~b}$ shows the maximum values for the range of $3 \leq \sigma \leq 10^{3}$. 


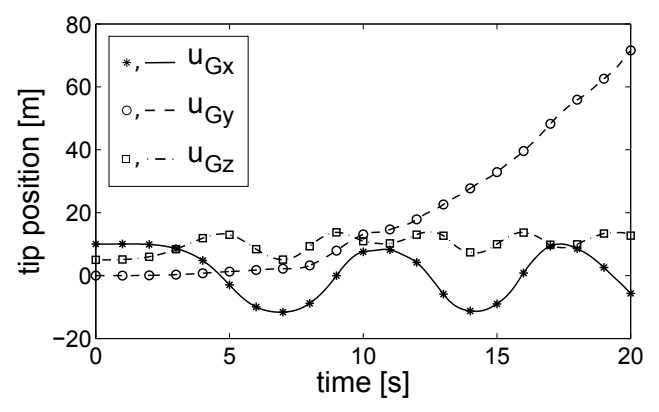

(a) Inertial positions of point $\mathrm{P}, u_{G}$.

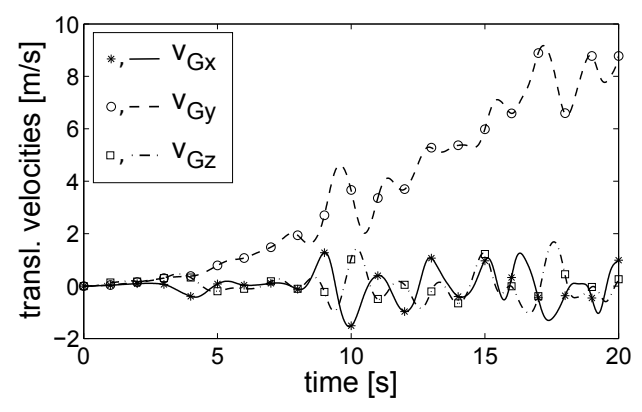

(b) Translational rigid-body velocity, $v_{G}$.

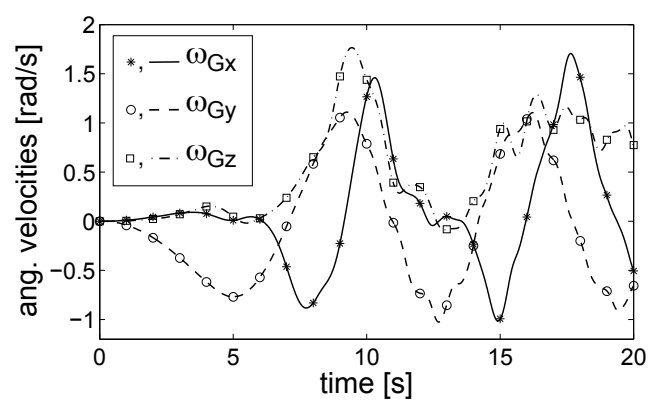

(c) Angular rigid-body velocity, $\omega_{G}$.

Figure 8: Comparison of FMB, load case 1, in SHARP (curves) with SAMCEF Mecano (markers).

Figure 11 presents the maximum relative error norm of the linear-flexible/nonlinearrigid formulation with respect to the fully-nonlinear solution in predicting rigid-body velocities and right tip deflection for a range of $\sigma$ values. The solid curves show that for very flexible cases with $\sigma<10$ and relative maximum tip displacements of $10-25 \%$ the dynamic response is nonlinear and cannot be captured by the linearised formulation with relative errors above $10 \%$. However, the error decreases exponentially with increasing values of $\sigma \geq 10$. The effect of nonlinearity on the elastic deformations for this problem is presented in detail in Section 4.3.1. The relative error of the rigid-body 

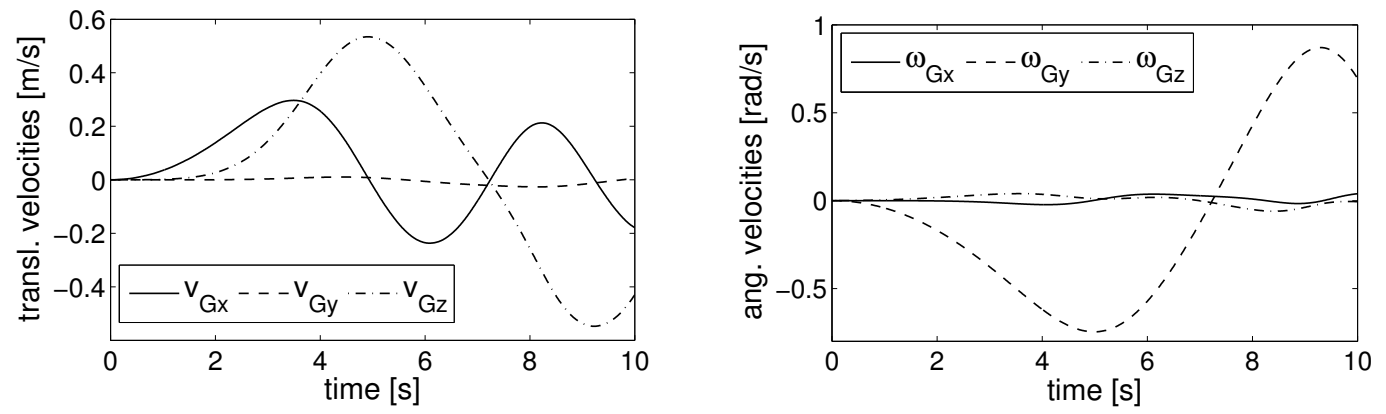

Figure 9: Rigid-body velocities of the FMB for load case 2. $[\sigma=10]$.
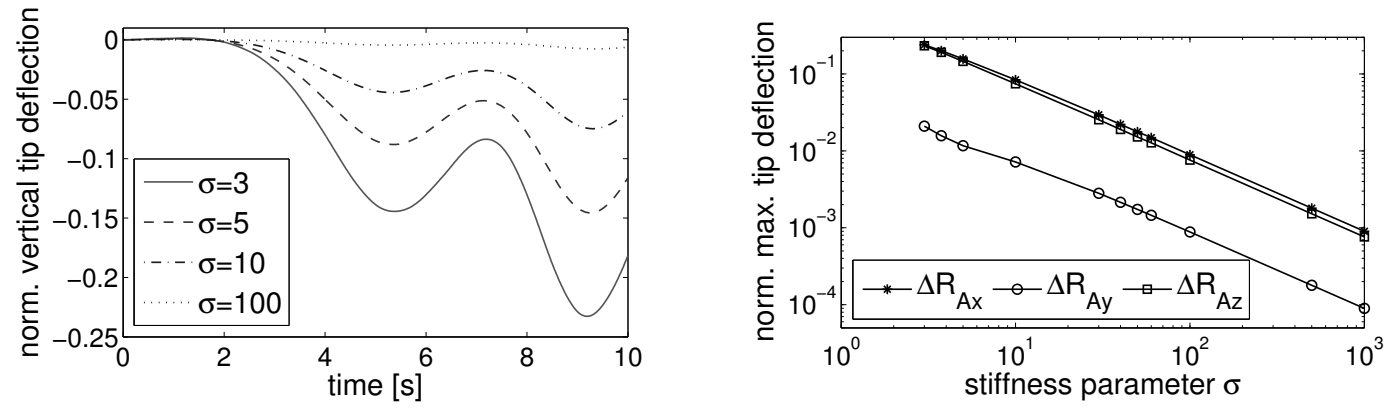

(a) Vertical displacement of Point $\mathrm{P}, \Delta R_{A z} / L_{2}$.

(b) Maximum tip deflection, $\max \left(\Delta R_{A} / L_{2}\right)$.

Figure 10: Tip deformations of FMB, load case 2, for varying stiffness parameter $\sigma$.

velocities, presented in Fig. 11a, shows that the relative error norm of the translational rigid-body velocities remains an order of magnitude higher than for the angular velocities. As the linearised solution assumes constant CM position with respect to frame $A$, the error in the angular velocities is compounded.

It is often assumed in dynamics problems that the mass matrix of the governing EoM is constant for small deformations [45]. The different effects of geometric non-linearities are analysed in the following by assuming constant mass matrix $\mathcal{M}\left(\eta_{0}\right)$ in the fullynonlinear formulation, but all other terms in Eq. (8) remain time-dependent. Figure 11 shows, superimposed with dashed curves, the relative error norm of this simplified- 


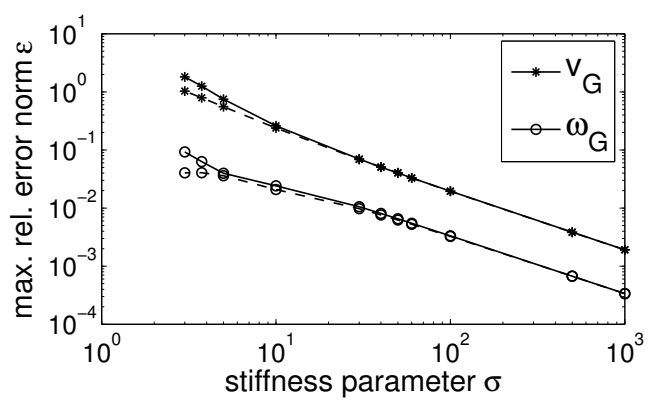

(a) Rigid-body velocities, $v_{G}$ and $\omega_{G}$.

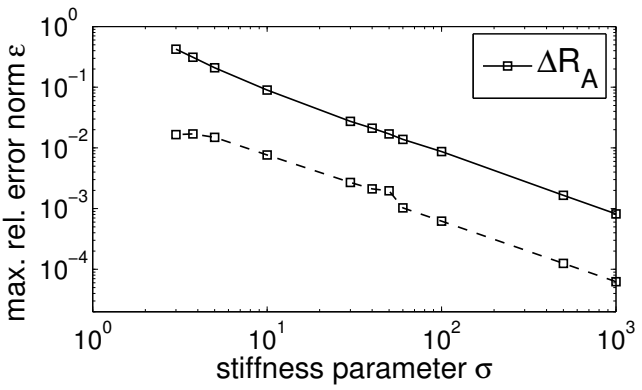

(b) Right tip displacement, $\Delta R_{A} / L_{2}$.

Figure 11: Linearisation of elastic DoF for FMB with load case 2. Maximum relative error norm of linear-flexible formulation (solid) and simplified nonlinear solution with constant mass matrix $\mathcal{M}=\mathcal{M}\left(\eta_{0}\right)$ (dashed) for different $\sigma$.

nonlinear formulation with respect to the fully-nonlinear solution. It suggests that for the analysed FMB problem within the linear-flexible regime $(\sigma \geq 10)$, the error in the rigid-body response is predominantly affected by assuming constant mass matrix $\mathcal{M}$. Only for very large deformations $(\sigma<10)$, it is possible to distinguish between the linear-flexible/nonlinear-rigid and simplified-nonlinear error norms in Fig. 11a. This is clearly not the case when predicting the tip deflection, $\Delta R_{A}$, shown in Fig. $11 \mathrm{~b}$, where linearisation of the elastic forces in addition to inertial forces has a detrimental effect. The relative error norm decreases exponentially in both cases, but the simplified nonlinear solution with $\mathcal{M}=\mathcal{M}\left(\eta_{0}\right)$ shows an irregularity in the range $30 \geq \sigma \geq 60$, where we included more data points to investigate this.

In summary, this section demonstrated the applicability of the implemented linearflexible/nonlinear-rigid formulation to stiff multi-beam configurations subject to small deformations and large rigid-body motions. Different sources of nonlinearity were identified to affect the dynamics of flexible bodies, where it was shown that changes in the 
global inertia tensor can have a strong effect on the rigid-body response of the structure.

\subsubsection{Modal Reduction of FMB}

In this section, projection of the nonlinear flexible-body dynamics equations with linearised elastic DoF on modes is explored. This is the application of the theory presented in Section 3.3, which forms the basis for the subsequent evaluation of the mean-axes approach. The problem at hand is the FMB with load case 2 and stiffness parameter $\sigma=10$, for which the linear-flexible/nonlinear-rigid solution proved to adequately predict angular rigid-body velocities and tip displacements with maximum relative errors of $2.4 \%$ and 9.0\%, respectively, as shown in Fig. 11 .

Table 3: First elastic modes for the FMB problem. $[\sigma=10]$

\begin{tabular}{ccl}
\hline Mode & $\omega[\mathrm{rad} / \mathrm{s}]$ & Description \\
\hline 1 & 6.92 & 1st symmetric in-plane bending \\
2 & 15.32 & 1st torsion \\
3 & 16.37 & 1st antisymmetric in-plane bending \\
4 & 21.58 & 1st symmetric out-of-plane bending \\
5 & 23.13 & 2nd symmetric in-plane bending \\
6 & 40.23 & 2nd antisymmetric in-plane bending \\
7 & 61.46 & 2nd torsion \\
8 & 67.91 & 3rd symmetric in-plane bending \\
\hline
\end{tabular}

Vibration modes are obtained for the unconstrained structure at the initial configuration by solving the eigenvalue problem posed by the unforced fully-linearised EoM, Eq. (22), with zero damping matrix. This results in $n$ elastic free-free modes and 6 rigidbody modes, where $n$ is the number of elastic DoF of the problem (here: $n=6 \cdot 120$ ). Table 3 presents the the first eight elastic modes with the mode shapes shown in Fig. 
12 and characterised by their symmetry properties. It is important to note that the $\mathrm{CM}$ position of these vibration modes remains constant.

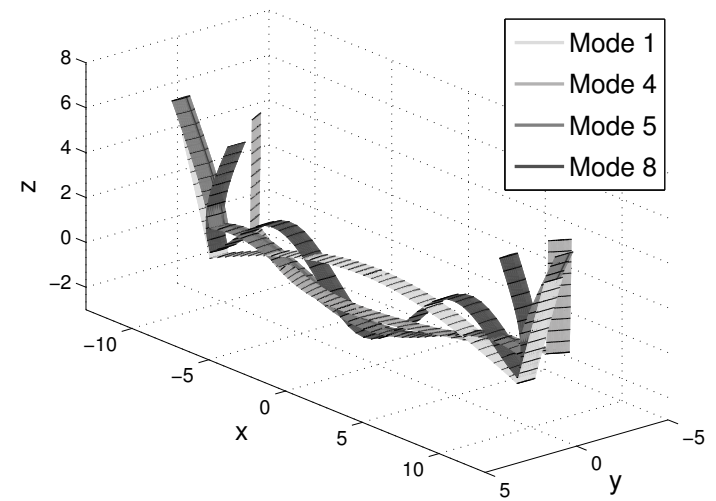

(a) Symmetric modes

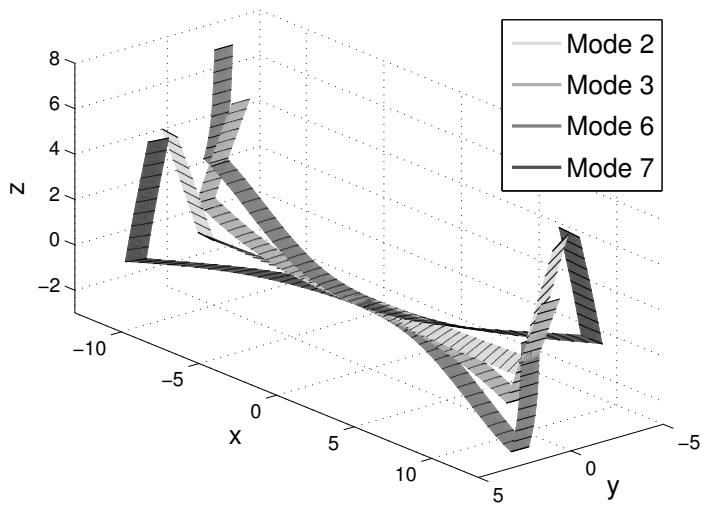

(b) Antisymmetric modes

Figure 12: First elastic mode shapes for the FMB problem. $[\sigma=10]$

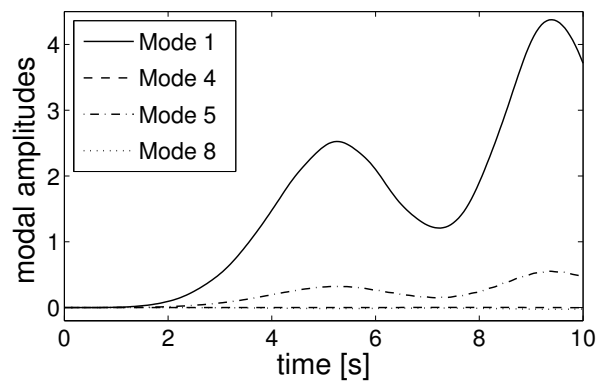

(a) Symmetric modes

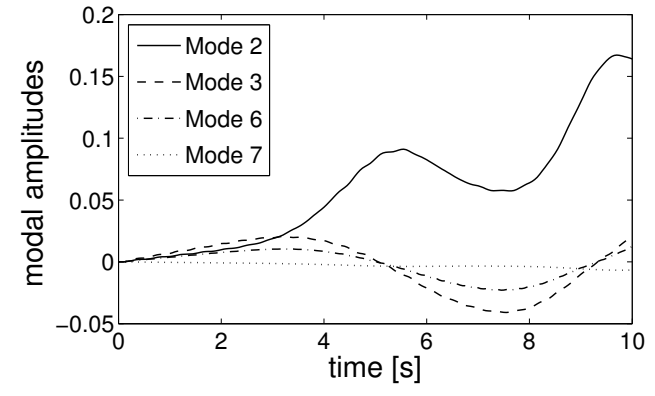

(b) Antisymmetric modes

Figure 13: Response of the first eight elastic modes for FMB, load case 2. $[\sigma=10]$

The dynamic problem is subsequently solved by projecting the linear-flexible/nonlinear-rigid dynamics Eq. 21) to modal coordinates. From the modal response of the FMB problem with load case 2 in Fig. 13 , it is clear that the dominant modes in the 
response are the first symmetric in-plane bending mode (Mode 1) and the first out-ofplane modes in torsion (Mode 2) and bending (Mode 4). This can also be seen in Fig. 14. which compares the rigid-body response and elastic deformations of the projected solution with linear-flexible/nonlinear-rigid results for varying number of elastic modes. With this approach it is possible to reduce the system size from 366 DoF to 16 modes (10 elastic modes and the six rigid-body modes) with sufficiently-small relative errors below $1 \%$. The system size can be further reduced by only including the modes which are dominant in predicting the response, as seen in Fig. 13. Figure 14 also shows the effect of flexibility on the rigid-body response of the structure for zero elastic modes resulting in maximum relative errors above $10 \%$.

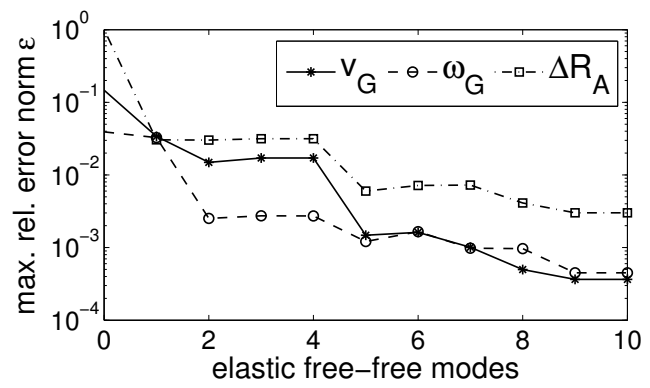

Figure 14: Modal projection of nonlinear flexible-body EoM with linearised elastic DoF for the FMB, load case 2. Maximum relative error norm of rigid-body velocities and right tip displacement against number of elastic modes. $[\sigma=10]$

Because of the linear and quadratic dependencies of the perturbed damping and stiffness matrices, $\overline{\mathcal{C}}$ and $\overline{\mathcal{K}}$ in Eq. (24), with rigid-body velocities, $\beta$, we write the modal system matrices in terms of constant tensors, $c$ and $k$, as shown in Eq. 25. Computing these tensors only once increases computational efficiency because of their sparse structure. This is illustrated in Table A in the Appendix only for the thirdorder damping tensor, $c$, but applies equally to the fourth-order stiffness tensor, $k$. The 
table only shows the first three elastic modes, which predict the response sufficiently with a maximum error of 3\% (see Fig. 14). With the description of Table A in the Appendix, it is possible to understand the contribution of the coupling terms between elastic modes and rigid-body motion on the gyroscopic forces, which are mostly affected by rigid-body rotary motion $(r=\{4,5,6\})$ as expected from the physical origin of the gyroscopic terms.

\subsection{An Assessment of the Mean-Axes Approximation}

The previous sections demonstrated a procedure for the consistent linearisation of the elastic DoF and subsequent projection onto modes under the assumption of small deformations but with large (nonlinear) rigid-body motion. However, due to the contribution of the gyroscopic forces to tangent damping and stiffness matrices in Eq. (19), the projected EoM still have coupling terms between the rigid-body and structural dynamics of the body. These coupling terms are often neglected for small elastic deformations by writing the EoM at the CM in terms of the mean-axes system, which is defined such that translational and angular momenta due to elastic deformations have zero contribution to the kinetic energy [12].

The mean-axes constraint is naturally enforced by projecting the linear EoM onto free-free modes [45]. Here, we obtain the free-free modes in the classical sense by solving the eigenvalue problem given by the unconstrained, undamped elastic EoM as, $\mathcal{M}^{S S} \ddot{\eta}^{f}+\mathcal{K}_{\text {stif }}^{S} \eta^{f}=0$, where $\eta^{f}$ is the vector of elastic DoF without enforcing the boundary condition of the clamped node at the origin of frame $A$. The corresponding mass matrix, $\mathcal{M}^{S S}$, is defined in Eq. (13) and the stiffness matrix, $\mathcal{K}_{\text {stif }}^{S}$, is obtained through linearisation of the elastic forces, $Q_{s t i f}^{S}$. The relative displacements of the

flexible body can then be described in terms of modes shapes, $\Phi^{f}$, and generalised displacements, $p^{f}$, such that $\eta^{f}=\sum_{i} \Phi_{i}^{f} p_{i}^{f}$. Due to orthogonality of the free-free modes 
and if we neglect the contribution of elastic deformations to the gyroscopic forces in Eq. (18), then it is possible to solve the mean-axes EoM,

$$
\ddot{p}_{i}^{f}+\omega_{i}^{2} p_{i}^{f}=\left(\Phi_{i}^{f}\right)^{\top} Q_{e x t}^{f}(\eta, \beta, \zeta)
$$

separately from the nonlinear rigid-body dynamics EoM, extracted from Eq. (18), as

$$
\mathcal{M}^{R R}(\eta) \dot{\beta}+\mathcal{C}^{R R}(\eta, \beta) \beta=Q_{e x t}^{R}(\eta, \beta, \zeta)
$$

where $\omega_{i}$ is the vibration frequency of the $i$ th mode. However, the resulting set of EoM can still be coupled if the external forces depend on the rigid-body velocities and orientation, $\beta$ and $\zeta$.

The effect of inertial decoupling between the elastic and rigid-body motion is analysed in this section first for the FMB problem with load case 2 in the range of stiffness parameters, $10 \leq \sigma \leq 10^{3}$, for which the linearised solution proved to be adequate, as shown in Fig. 11. In the second study the mean-axes assumption is investigated on a more complex problem, where the FMB enters a spiral motion to investigate the effect of simultaneous translational and angular rigid-body motion. For both problems, the decoupled mean-axes solution is compared to the linear-flexible/nonlinear-rigid and fully-nonlinear solution, to understand the effect of neglecting the coupling terms due to the gyroscopic nature. Enough modes were included in the mean-axes solutions until convergence was reached.

\subsubsection{FMB in Rotation (load case 2)}

For the FMB with load case 2, Fig. 15 shows the relative error norm of the meanaxes approach in predicting the rigid-body response and deflection of point $\mathrm{P}$ for a range of $\sigma$ values. To be able to compare to the mean-axes solution, the rigid-body motion is computed in this problem at the CM of the initially undeformed geometry. 


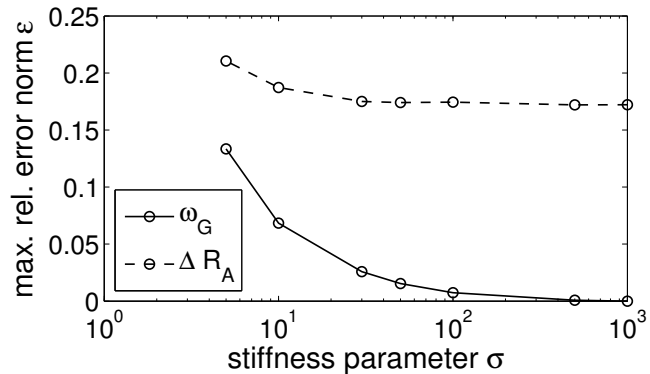

Figure 15: Maximum relative error norm of rigid-body angular velocities and right tip displacement of mean-axes solution for the FMB, load case 2, compared to linearflexible/nonlinear-rigid for different values of $\sigma .[\Delta t=0.01 \mathrm{~s}$ and 1202 -noded elements $]$

Hence, only angular velocities are included in Fig. 15. The results indicate that for small deformations with $\sigma>30$ and relative tip deflections below $2 \%$, the mean-axes model predicts the rigid-body response of this problem with relative errors below $5 \%$. However, it is obvious from the figure that neglecting the coupling terms results in a constant error of about $17 \%$ for the elastic deformations over the same range of stiffness parameters.

To understand this effect, Fig. 16 shows the normalised right tip deflections for a range of stiffness values to compare the fully-nonlinear (Eq. (8)), linearised (Eq. (22)) and mean-axes solutions (Eq. (27)). The linearised formulation cannot capture the hardening effect due to large deformations which results in over-predicting the elastic response. However, it is clear that application of the mean-axes constraint results in smaller deflections independent of the material stiffness, which happens to match the nonlinear solution closer for the very flexible case $(\sigma=5)$. Under the mean-axes assumption, the gyroscopic terms in the structural dynamics EoM, defined in Eqs. (18)-(19), are neglected. For large values of $\sigma$, the rigid-body dynamics of the structure 


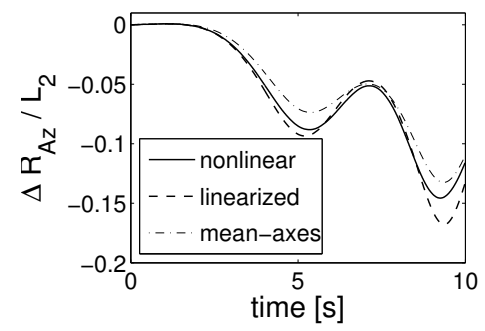

(a) $\sigma=5$

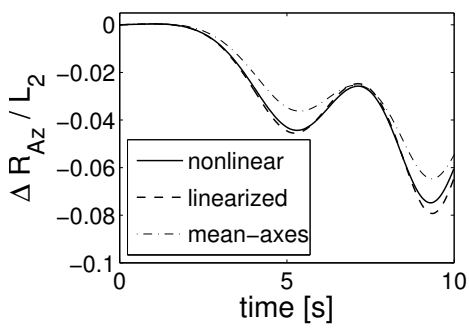

(b) $\sigma=10$

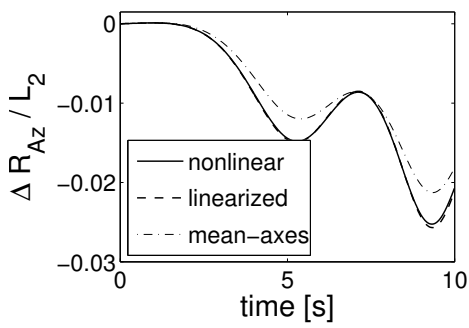

(c) $\sigma=30$

Figure 16: Comparison of fully-nonlinear, linear-flexible/nonlinear-rigid and mean-axes approach in predicting the vertical tip displacement of Point $\mathrm{P}$ in Fig. 6 for the FMB problem with load case 2 and different $\sigma$ values.

do not change with increasing stiffness, which implies that the gyroscopic damping and stiffness matrices, $\overline{\mathcal{C}}^{S S}, \overline{\mathcal{C}}^{S R}$ and $\overline{\mathcal{K}}_{g y r}^{S}$, are independent of $\sigma$. Whereas the phase difference in the elastic response between the mean-axes and nonlinear solutions is evident for small values of $\sigma$, for stiff problems $(\sigma \geq 30)$ the maximum relative error norm occurs at the $t=9.35 \mathrm{~s}$, which explains why the relative contribution of the gyroscopic forces to the structural dynamics remains constant with $\sigma$.

\subsubsection{FMB in Spiral Motion}

This final study is motivated by dynamic stability problems of aircraft. The geometry and material properties of the FMB are defined in Fig. 6. A set of follower forces and moments is now acting at the CM of the initially undeformed geometry, which is rigidly-linked to the frame at point $P_{G}$, as shown in Fig. 17. This implies that, under deformations of the structure, the origin of loading may not remain at the CM. The follower loading is applied as a ramp with time $t$ such that the FMB follows a circular rigid-body motion with radius $R_{S}=80 \mathrm{~m}$, where $m$ is the total mass of the frame and $J_{z}$ is the (constant) moment of inertia around the $z$ axis at the reference 
configuration. An additional dead load is applied at an offset at point $P_{G}$, shown in Fig. 17, to stabilise the structure, as the FMB tends to diverge from the spiral path under deformation. The stiffness parameter $\sigma$ is used again in this problem to vary the stiffness of the structure. A mesh size of 60 2-noded elements and a time step of $\Delta t=0.1 \mathrm{~s}$ was found to be sufficient to capture the predominantly rigid-body dynamics of the problem simulated for $80 \mathrm{~s}$.

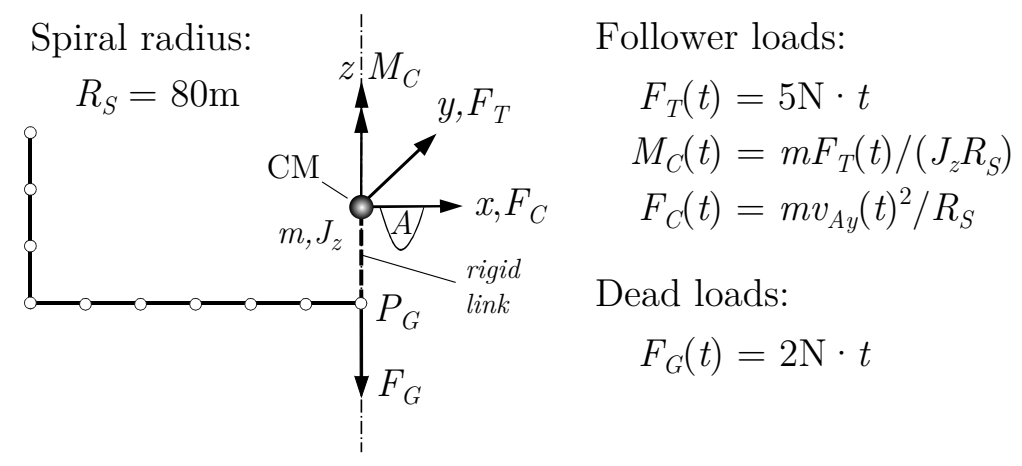

Figure 17: Load history of the FMB spiral problem.

The overall motion of the centroid is shown in Fig. 18 for a range of stiffness parameters $\sigma$ with the corresponding vertical tip deflections presented in Fig. 19. The effect of large deformations on the rigid-body motion is captured in Fig. 18a, which shows large discrepancies between the linearised and fully-nonlinear solution. With increasing values of $\sigma$ the two solutions converge, such that for $\sigma \geq 10$ the linearised formulation can accurately capture the very large and nonlinear rigid-body motion of the structure accurately with relative errors below 3.2\%. However, the overall motion is still affected by the (small) elastic deformations. This is shown by including the trajectory of the rigid structure in Fig. 18 to identify possible values of $\sigma$ where the mean-axes approach can be applied. It can be seen that the spiral motion of the FMB 


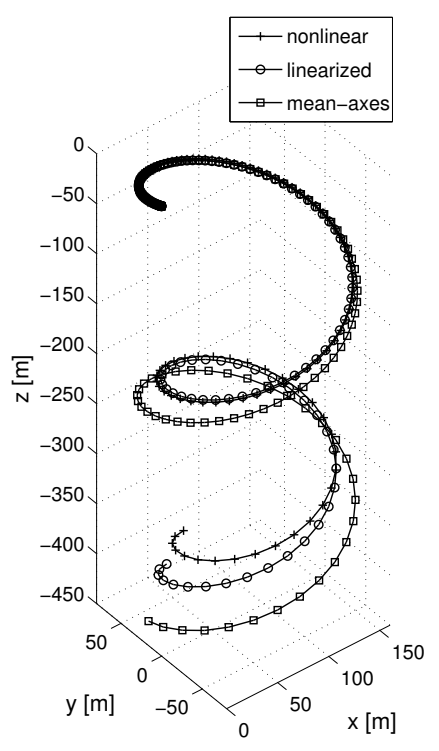

(a) $\sigma=4$

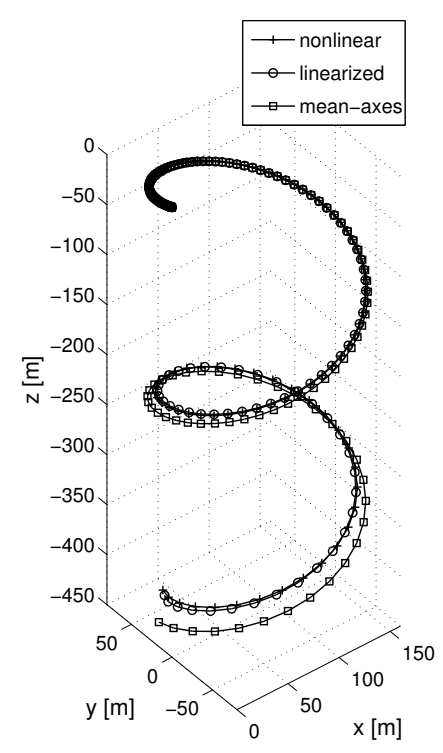

(b) $\sigma=10$

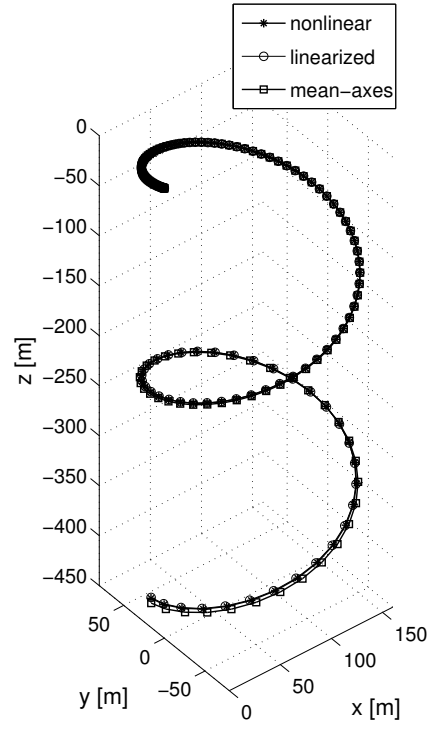

(c) $\sigma=50$

Figure 18: Trajectory of the FMB spiral problem for the different formulations. $[\Delta t=$ $0.1 \mathrm{~s}$ and 60 2-noded elements]

converges to the rigid case for $\sigma \geq 50$ with relative errors below $1 \%$.

Figure 19 shows the elastic deformations of the FMB comparing the fully-nonlinear solution with the linearised formulation and the mean-axes approach. As expected, the linearised formulation tends to over-predict the structural deformations in the very flexible case with $\sigma=4$, where geometrical nonlinearity effects are dominant. However, the elastic response is becoming linear with increasing stiffness of the structure. Neglecting the nonlinear gyroscopic forcing terms in the mean-axes approach counteracts this over-prediction and happens to match the nonlinear solution more accurately for the flexible range $(\sigma<10)$, even though the rigid-body response is still largely affected by the elastic deformations in this range, as shown in Fig. 18a. 


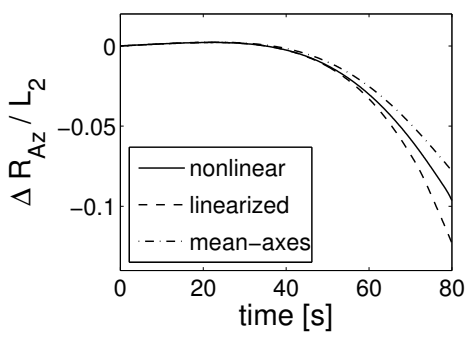

(a) $\sigma=4$

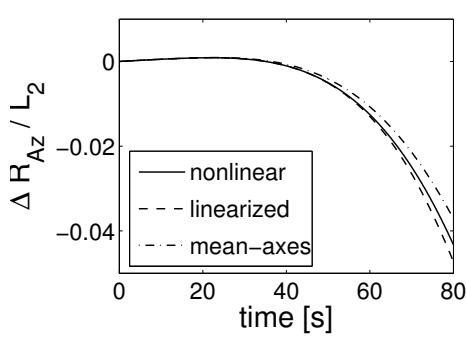

(b) $\sigma=10$

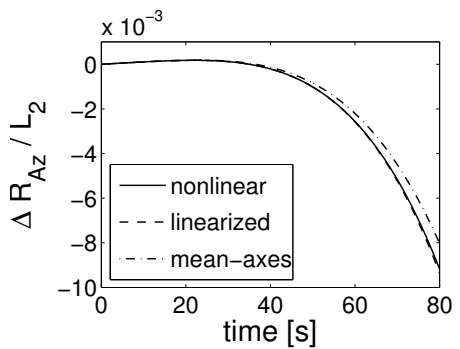

(c) $\sigma=50$

Figure 19: Comparison of fully-nonlinear, linear-flexible/nonlinear-rigid and mean-axes approach in predicting vertical tip displacement of point P in Fig. 6 for the FMB spiral problem and different $\sigma$ values. $[\Delta=0.1 \mathrm{~s}$ and 60 2-noded elements]

The same can be observed in Fig. 20, which shows the relative error norm of linearflexible/nonlinear-rigid and mean-axes approximations compared to the fully-nonlinear solution in predicting the maximum tip displacement of point $\mathrm{P}, \max \left(\Delta R_{A}\right)$, for a range of $\sigma$ values. The mean-axes approach neglects the couplings and under-predicts elastic deformations, which results in smaller errors compared to the linearised solution for very flexible cases $(\sigma<8)$. However, this result is problem-specific and for stiff cases $(\sigma>20)$, the relative error norm of the mean-axes assumption converges to a constant value of about $12 \%$. As before, when $\sigma \rightarrow \infty$, the relative contribution of the elastic gyroscopic forces to the structural dynamics becomes a function merely of $\sigma$ resulting in constant relative errors in the displacements.

\section{Conclusions}

This paper has presented a derivation of the equations of motion of flexible-body dynamics for small deformations and large rigid-body motions around a nonlinear static equilibrium configuration. This situation is relevant for the dynamic analysis of flexible 


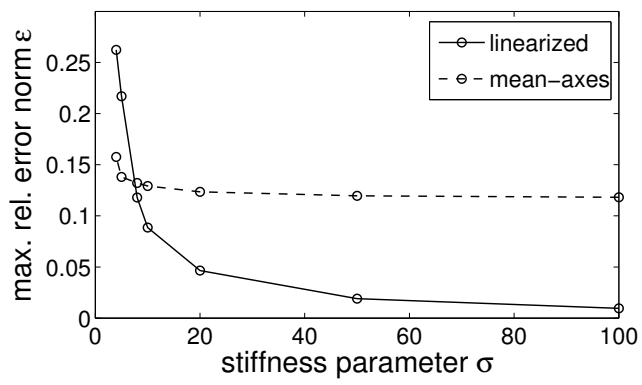

Figure 20: Relative error norms of maximum tip displacements at Point P, $\max \left(\Delta R_{A z} / L_{2}\right)$, for FMB spiral problem comparing linear-flexible/nonlinear-rigid and mean-axes approximations to fully-nonlinear solution. $[\Delta=0.1 \mathrm{~s}$ and 602 -noded elements]

aircraft with large-aspect-ratio wings, but the formulation in this work is a generic one.

The resulting set of dynamics equations has been written in matrix form, in which the matrix coefficients have only a quadratic dependency on the body velocities. In a time-marching solution, this implies that the coefficients have to be computed as the body-velocity changes, but the equations are still linear in the structural degrees of freedom. A very large reduction in problem size can then be obtained if the FEM discretisation is approximated by a few vibration modes of the unconstrained structure. This was further exploited by obtaining closed-form expressions for all the coefficients in the equations that only need to be computed once. The resulting modal form of the equations of motion can capture all couplings between the nonlinear rigid-body dynamics and the linearised structural dynamics due to gyroscopic effects. These coupling terms are neglected in the mean-axes approach typically used in flexible aircraft flight dynamics.

Numerical studies presented in this work exercise this modal approach on a complex multi-beam configuration. It was found that writing the system equations in tensor 
form allows the easy identification of important coupling terms between different elastic modes and the rigid-body motion (in the example, it was the coupling between yaw rate and in-plane bending modes which results in large gyroscopic forces). Results also served to evaluate the validity of the mean-axes approximation. Whereas the rigidbody response always converges to the rigid case for very stiff problems, the mean-axes approach fails to predict the elastic deformations accurately if the problem is of a rotational nature. This result was shown, both analytically and numerically, to be independent of the level of deformation.

In summary, a consistent linearisation of the structural deformations in the dynamic analysis of flexible bodies can be introduced for small deformations which captures all coupling terms due to gyroscopic effects. This provides a more accurate description of the dynamics of relatively stiff structures than the mean-axes approach with only a small additional computational cost.

\section{Acknowledgements}

The work of Henrik Hesse is sponsored by the UK Engineering and Physical Sciences Research Council (EPSRC). This support is gratefully acknowledged.

\section{References}

[1] C. E. Cesnik, D. H. Hodges, VABS: A new concept for composite rotor blade cross-sectional modeling, Journal of the American Helicopter Society 42 (1997) 27.

[2] N. Buannic, P. Cartraud, Higher-order effective modeling of periodic heterogeneous beams. I. asymptotic expansion method, International Journal of Solids and Structures 38 (2001) 7139 - 7161 . 
[3] R. Palacios, C. Cesnik, Cross-sectional analysis of non-homogeneous anisotropic active slender structures, AIAA Journal 43 (12) (2005) 2624-2638.

[4] G. J. Kennedy, J. R. Martins, A homogenization-based theory for anisotropic beams with accurate through-section stress and strain prediction, International Journal of Solids and Structures 49 (1) (2012) $54-72$.

[5] V. Giavotto, M. Borri, P. Mantegazza, G. Ghiringhelli, V. Carmaschi, G. C. Maffioli, F. Mussi, Anisotropic beam theory and applications, Computers \& Structures 16 (1-4) (1983) 403-413.

[6] D. H. Hodges, A mixed variational formulation based on exact intrinsic equations for dynamics of moving beams, International Journal of Solids and Structures 26 (11) (1990) 1253-1273.

[7] D. H. Hodges, Geometrically exact, intrinsic theory for dynamics of curved and twisted anisotropic beams, AIAA Journal 41 (6) (2003) 1131-1137.

[8] O. A. Bauchau, Flexible Multibody Dynamics, Springer, London, UK, 2010.

[9] D. H. Hodges, Nonlinear Composite Beam Theory, Progress in Astronautics \& Aeronautics, Vol 213, American Institue of Aeronautics and Astronautics, Reston, Virginia, USA, 2006.

[10] D. J. Lucia, The sensorcraft configurations: A non-linear aeroservoelastic challenge for aviation, in: Proceedings of the 46th AIAA/ASME/ASCE/AHS/ASC Structures, Structural Dynamics and Materials Conference, AIAA Paper 2005-1943, Austin, TX, 2005. 
[11] R. Cook, R. Palacios, P. J. Goulart, I. Roberts, Robust Manoeuvring and Gust Alleviation of Very Flexible Aircraft using Novel Control Effectors, in: Proceedings of the 2011 International Forum of Aeroelasticity and Structural Dynamics (IFASD), Paper No 2011-047, Paris, France, 2011.

[12] R. D. Milne, Dynamics of the deformable plane, Tech. Rep. 3345, Her Majesty's Stationary Office, London, UK (1962).

[13] O. P. Agrawal, A. A. Shabana, Application of deformable-body mean axis to flexible multibody system dynamics, Computer Methods in Applied Mechanics and Engineering 56 (2) (1986) 217-245.

[14] P. P. Friedmann, J. J. McNamara, B. J. Thuruthimattam, I. Nydick, Aeroelastic analysis of hypersonic vehicles, Journal of Fluids and Structures 19 (5) (2004) $681-712$.

[15] T. Kier, G. Looye, M. Scharpenberg, M. Reijerkerk, Process, methods and tools for flexible aircraft flight dynamics model integration, in: Proceedings of International Forum on Aeroelasticity and Structural Dynamics (IFASD), Stockholm, Sweden, 2007.

[16] F. Silvestre, R. Luckner, Integrated Model for the Flight Mechanics of a Flexible Aircraft in the Time Domain, in: Proceedings of the International Forum of Aeroelasticity and Structural Dynamics (IFASD), Seattle, Washington, USA, 2009.

[17] L. Meirovitch, I. Tuzcu, The lure of the mean axes, Journal of Applied Mechanics 74 (3) (2007) 497-504.

[18] L. Meirovitch, A new modal method for the response of structures rotating in space, Acta Astronautica 2 (7-8) (1975) 563576. 
[19] L. Meirovitch, J. N. Juang, Dynamics of a gravity-gradient stabilized flexible spacecraft, Tech. rep., NASA CR-2456 (1974).

[20] L. Meirovitch, Hybrid state equations of motion for flexible bodies in terms of quasicoordinates, Journal of Guidance, Control, and Dynamics 14 (5) (1991) 1008-1013.

[21] A. E. H. Love, A Treatise on the Mathematical Theory of Elasticity, 4th Edition, Dover Publications Inc, New York, NY, USA, 1944.

[22] E. Reissner, On one-dimensional large-displacement finite-strain beam theory, Studies in Applied Mathematics 52 (1973) 87-95.

[23] J. Simo, A finite strain beam formulation. the three-dimensional dynamic problem. Part I, Computer Methods in Applied Mechanics and Engineering 49 (1) (1985) $55-70$.

[24] J. C. Simo, L. Vu-Quoc, A three-dimensional finite-strain rod model. Part II: computational aspects, Computer Methods in Applied Mechanics and Engineering 58 (1) (1986) 79-116.

[25] J. C. Simo, L. Vu-Quoc, On the Dynamics of Flexible Beams Under Large Overall Motions - The Plane Case: Part II, Journal of Applied Mechanics 53 (4) (1986) $855-863$.

[26] J. C. Simo, L. Vu-Quoc, On the dynamics in space of rods undergoing large motions - A geometrically exact approach, Computer Methods in Applied Mechanics and Engineering 66 (2) (1988) 125-161.

[27] A. Cardona, M. Géradin, A beam finite element non-linear theory with finite rotations, International Journal for Numerical Methods in Engineering 26 (11) (1988) $2403-2438$. 
[28] M. Géradin, A. Cardona, Flexible multibody dynamics: a finite element approach, John Wiley and Sons, Inc., Chichester, UK, 2001.

[29] L. Meirovitch, I. Tuzcu, Unified theory for the dynamics and control of maneuvering flexible aircraft, AIAA Journal 42 (4) (2004) 714-727.

[30] J. Murua, R. Palacios, J. M. R. Graham, Assessment of wake-tail interference effects on the dynamics of flexible aircraft, AIAA Journal [Accepted for publication].

[31] M. A. Crisfield, G. Jelenić, Objectivity of strain measures in the geometrically exact three-dimensional beam theory and its finite-element implementation, Proceedings of the Royal Society of London. Series A: Mathematical, Physical and Engineering Sciences 455 (1999) 1125-1147.

[32] O. A. Bauchau, A. Epple, S. Heo, Interpolation of finite rotations in flexible multibody dynamics simulations, Proceedings of the Institution of Mechanical Engineers, Part K: Journal of Multi-body Dynamics 222 (4) (2008) 353-366.

[33] B. L. Stevens, F. L. Lewis, Aircraft Control and Simulation, John Wiley \& Sons, Inc., New York, NY, USA, 1992.

[34] J. C. Simo, N. Tarnow, M. Doblare, Non-linear dynamics of three-dimensional rods: Exact energy and momentum conserving algorithms, International Journal for Numerical Methods in Engineering 38 (9) (1995) 1431-1473.

[35] O. Bauchau, N. Theron, Energy decaying scheme for non-linear beam models, Computer Methods in Applied Mechanics and Engineering 134 (12) (1996) 37-56.

[36] M. Géradin, D. Rixen, Mechanical Vibrations: Theory and Application to Structural Dynamics, 2nd Edition, Wiley-Blackwell, 1997. 
[37] S. Krenk, Energy conservation in Newmark based time integration algorithms, Computer Methods in Applied Mechanics and Engineering 195 (44-47) (2006) 6110-6124.

[38] K.-J. Bathe, Finite-Element Procedures, Prentice-Hall Inc, Upper Saddle River, New Jersey, USA, 1996.

[39] R. Palacios, J. Murua, R. Cook, Structural and Aerodynamic Models in the Nonlinear Flight Dynamics of Very Flexible Aircraft, AIAA Journal 48 (11) (2010) $2648-2559$.

[40] M. Gams, M. Saje, S. Srpčič, I. Planinc, Finite element dynamic analysis of geometrically exact planar beams, Computers \& Structures 85 (17-18) (2007) 1409-1419.

[41] K. Hsiao, J. Jang, Dynamic analysis of planar flexible mechanisms by co-rotational formulation, Computer Methods in Applied Mechanics and Engineering 87 (1) (1991) $1-14$.

[42] K. M. Hsiao, J. Y. Lin, W. Y. Lin, A consistent co-rotational finite element formulation for geometrically nonlinear dynamic analysis of 3-D beams, Computer Methods in Applied Mechanics and Engineering 169 (1-2) (1999) 1-18.

[43] M. Iura, S. N. Atluri, On a consistent theory, and variational formulation of finitely stretched and rotated 3-D space-curved beams, Computational Mechanics 4 (2) (1988) 73-88.

[44] K.-J. Bathe, G. Noh, Insight into an implicit time integration scheme for structural dynamics, Computers \& Structures 98-99 (2012) 1-6.

[45] M. R. Waszak, D. K. Schmidt, Flight dynamics of aeroelastic vehicles, Journal of Aircraft 25 (6) (1988) 563-571. 


\section{Appendix A}

In Section 3.3, the nonlinear modal damping and stiffness matrices, $\Phi_{i j} \overline{\mathcal{C}}_{j k} \Phi_{k l}$ and $\Phi_{i j} \overline{\mathcal{K}}_{j k} \Phi_{k l}$, respectively, are written in terms of constant third and fourth-order tensors, $c_{i l r}$ and $k_{i l r s}$, defined in Eq. (25). Here, we explore the coupling terms of $c_{i l r}$ for the FMB problem defined in Fig. 6, with the effect of elastic and rigid-body velocities on the elastic state, $c_{r}^{S S}$ and $c_{r}^{S R}$, respectively, and the effect on the rigid-body gyroscopic forces due to elastic excitation, $c_{r}^{R S}$. The remaining terms, $c_{r}^{R R}$, originate from the gyroscopic terms of the standard nonlinear rigid-body dynamics part of the structure and are not of interest here. Table A shows the coupling terms of $c_{i l r}$ for the first 9 modes (3 elastic and 6 rigid-body) with

$$
\begin{gathered}
c_{r}^{S S}=\left\{c_{i l r} \mid(i=7 . .9) \wedge(l=7 . .9)\right\} \\
c_{r}^{S R}=\left\{c_{i l r} \mid(i=7 . .9) \wedge(l=1 . .6)\right\} \\
c_{r}^{R S}=\left\{c_{i l r} \mid(i=1 . .6) \wedge(l=7 . .9)\right\},
\end{gathered}
$$

where modes 1-6 are rigid-body modes and 7-9 the remaining elastic modes, which are ordered such that the lower frequencies occur first (e.g. first bending mode for $i, l=7$ ). Table A: Perturbed modal damping matrix in tensor form, $c_{i l r}$, for the FMB problem using the first three elastic modes in addition to the six rigid-body modes. $[\sigma=10]$. 


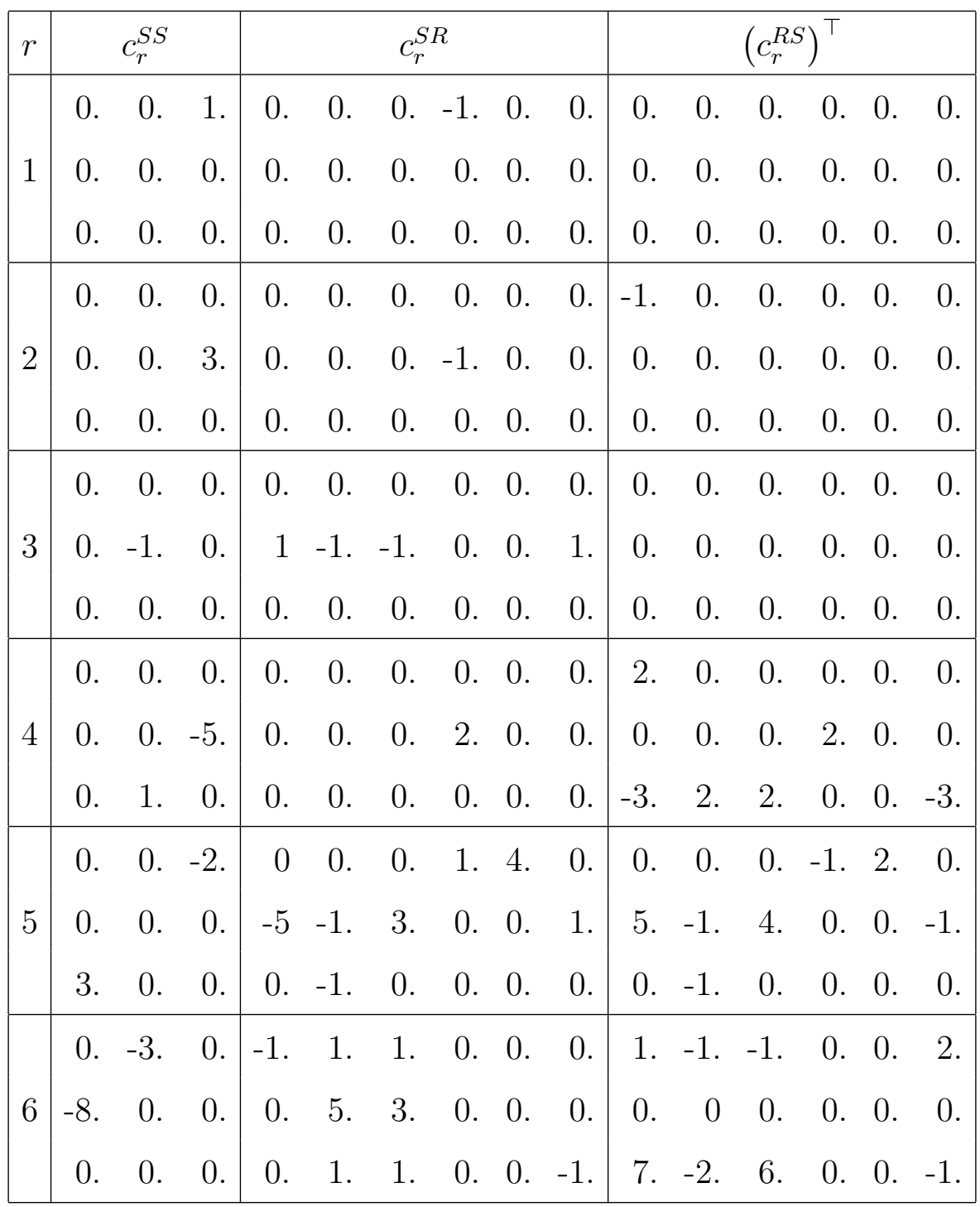

